\title{
ALGEBRAIC FOURIER RECONSTRUCTION OF PIECEWISE SMOOTH FUNCTIONS
}

\author{
DMITRY BATENKOV AND YOSEF YOMDIN
}

\begin{abstract}
Accurate reconstruction of piecewise smooth functions from a finite number of Fourier coefficients is an important problem in various applications. This problem exhibits an inherent inaccuracy, in particular, the Gibbs phenomenon, and it has been intensively investigated during the last few decades. Several nonlinear reconstruction methods have been proposed in the literature, and it is by now well-established that the "classical" convergence order can be completely restored up to the discontinuities. Still, the maximal accuracy of determining the positions of these discontinuities remains an open question.

In this paper we prove that the locations of the jumps (and subsequently the pointwise values of the function) can be reconstructed with at least "half the classical accuracy". In particular, we develop a constructive approximation procedure which, given the first $k$ Fourier coefficients of a piecewise $C^{2 d+1}$ function, recovers the locations of the jumps with accuracy $\sim k^{-(d+2)}$, and the values of the function between the jumps with accuracy $\sim k^{-(d+1)}$ (similar estimates are obtained for the associated jump magnitudes). A key ingredient of the algorithm is to start with the case of a single discontinuity, where a modified version of one of the existing algebraic methods (due to K. Eckhoff) may be applied. It turns out that the additional orders of smoothness produce highly correlated error terms in the Fourier coefficients, which eventually cancel out in the corresponding algebraic equations. To handle more than one jump, we apply a localization procedure via a convolution in the Fourier domain, which eventually preserves the accuracy estimates obtained for the single jump. We provide some numerical results which support the theoretical predictions.
\end{abstract}

\section{INTRODUCTION}

Consider the problem of reconstructing a function $f:[-\pi, \pi] \rightarrow \mathbb{R}$ from a finite number of its Fourier coefficients:

$$
c_{k}(f) \stackrel{\text { def }}{=} \frac{1}{2 \pi} \int_{-\pi}^{\pi} f(t) \mathrm{e}^{-\imath k t} \mathrm{~d} t, \quad k=0,1, \ldots, M
$$

It is well known that for periodic smooth functions, the truncated Fourier series

$$
\mathfrak{F}_{M}(f) \stackrel{\text { def }}{=} \sum_{|k|=0}^{M} c_{k}(f) \mathrm{e}^{\imath k x}
$$

Received by the editor May 13, 2010 and, in revised form, October 25, 2010.

2010 Mathematics Subject Classification. Primary 42A16; Secondary 65T40, 41A25.

Key words and phrases. Fourier inversion, nonlinear approximation, piecewise smooth functions.

We would like to thank Ch. Fefferman, E. Tadmor and N. Zobin for useful discussions.

This research was supported by ISF grant 264/09 and the Minerva Foundation.

(C)2011 American Mathematical Society 
converges to $f$ very fast, subsequently making Fourier analysis very attractive in a vast number of applications. We have by the classical Lebesgue lemma (see e.g. [34]) that

$$
\max _{-\pi \leq x \leq \pi}\left|f(x)-\mathfrak{F}_{M}(f)(x)\right| \leq(3+\ln M) \cdot E_{M}(f)
$$

where $E_{M}(f)$ is the error of the best uniform approximation to $f$ by trigonometric polynomials of degree at most $M$. This number, in turn, depends on the smoothness of the function. In particular:

(1) If $f$ is $d$-times continuously differentiable (including at the endpoints) and $\left|f^{(d)}(x)\right| \leq R$, then (see [46, Vol. I, Chapter 3, Theorem 13.6])

$$
E_{M}(f) \leq C_{d} \cdot R \cdot M^{-d} \text {. }
$$

(2) If $f$ is analytic, then by classical results of S. Bernstein (see e.g. 34, Chapter IX]) there exist constants $C$ and $q<1$ such that

$$
E_{M}(f) \leq C \cdot q^{M}
$$

Yet many realistic phenomena exhibit discontinuities, in which case the unknown function $f$ is only piecewise smooth. As a result, the trigonometric polynomial $\mathfrak{F}_{M}(f)$ no longer provides a good approximation to $f$ due to the slow convergence of the Fourier series (one of the manifestations of this fact is commonly known as the "Gibbs phenomenon"). It has very serious implications, for example, when using spectral methods to calculate solutions of PDEs with shocks. Therefore an important question arises: "Can such piecewise smooth functions be reconstructed from their Fourier measurements, with accuracy which is comparable to the 'classical' one (such as (1.1) or (1.2) )"?

This problem has received much attention, especially in the last few decades $(3,4,4,18,9,10,11,13,15,16,18,22,24,25,27,29,30,33,38,39,41,44$, would be only a partial list). It has long been known that the key problem for Fourier series acceleration is the detection of the shock locations. By now it is well-established that classical convergence rates can be restored uniformly up to the discontinuities (see e.g. 24]), but the corresponding question for the jump locations themselves is still open. Notice that any linear approximation procedure with free (a priori unknown) jump locations will not be able to achieve accuracy higher than $\frac{1}{\sqrt{M}}$; see 19 .

Several partial results and conjectures in this direction are known, in particular, the following. The concentration method of Gelb and Tadmor [18, 21, 41] recovers the jumps with first order accuracy, and it can be extended to higher orders. Kvernadze [29, 30] proves that his method can recover jumps of a $C^{3}$ function with second order accuracy. In [7, 19] we have conjectured that the locations of the jumps of a piecewise $C^{d}$ function can be recovered with accuracy $k^{-d}$ from its first $k$ Fourier coefficients (a similar conjecture is made in [43]). Both Eckhoff [15] and Banerjee and Geer [3] made the same conjectures with respect to their particular reconstruction methods. We would also like to mention a related but different problem: reconstruction of piecewise smooth functions from point measurements. There, adaptive approximations can achieve asymptotic accuracy $k^{-d}$ for piecewise $C^{d}$ functions [2, 35, 31].

With this motivation, our main goal in this paper is to arrive at a better understanding of the "optimal", or the "best possible" accuracy of reconstruction, especially with respect to the locations and the magnitudes of the jumps. As a 
means to achieve this goal, we develop a reconstruction method which allows for explicit accuracy analysis. Our method is a "hybrid" between a Fourier filtering technique which is first applied to localize the jumps, and the algebraic approach of Eckhoff and Kvernadze which is used in order to resolve each discontinuity one at a time to a high order of accuracy. It is precisely this "localization" which makes the subsequent analysis tractable.

Our accuracy analysis is "asymptotic" in nature, although we provide the explicit constants at every step. These constants in general depend upon various a priori estimates (such as the minimal distance between the jumps, or the upper bound on the jump magnitudes), which are presumably available. See the discussion in Section 2 below, in particular (2.4).

Let us now give a brief summary of the main results.

(1) If a function with a single jump has at least $2 d+1$ continuous derivatives everywhere except the jump, then the jump location can be recovered from the first $M$ Fourier coefficients with error at most $\sim M^{-d-2}$ (Theorem 4.13). In addition, a jump in the $l$-th derivative can be recovered with error at most $\sim M^{l-d-1}$ (Theorem 4.21). A key observation in the analysis is that the additional orders of smoothness produce highly correlated error terms in the Fourier coefficients, which eventually cancel out in the corresponding algebraic equations.

(2) The localization step does not "destroy" the above accuracy estimates (Theorem 5.2). Thus, the pointwise values of $f$ are recovered with the accuracy $\sim M^{-d-1}$ (Theorem 6.1) up to the jumps.

(3) Numerical simulations are consistent with the theoretical accuracy predictions (Section 7).

By means of this constructive approximation procedure with provable asymptotic convergence properties, we therefore demonstrate that the algebraic reconstruction methods for piecewise smooth data can be at least "half accurate" compared to the classical approximation theory for smooth data.

We provide an overview of the reconstruction procedure in Section 2, For expository reasons, the details of the localization step and the analysis of its accuracy are postponed until Section 5 . The resolution method of a single jump is presented in Section 3, while Section 4 is devoted to proving its asymptotic convergence order. Finally, the accuracy of the whole reconstruction is analyzed in Section 6 and some numerical results are presented in Section 7 Some common notations used throughout the paper are summarized below in Subsection 1.1

\subsection{Notation.}

- $\mathbb{N}$ denotes the natural numbers, $\mathbb{R}$ - the real numbers, $\mathbb{C}$ - the complex numbers.

- $C^{d}$ denotes the class of smooth functions which are continuously differentiable $d$ times everywhere. $C^{\infty}$ is the class of smooth functions having continuous derivatives of all orders.

- $B_{r}(z)$ is the ball of radius $r$ centered at $z$, and $\partial B_{r}(z)$ is the boundary of such a ball. 


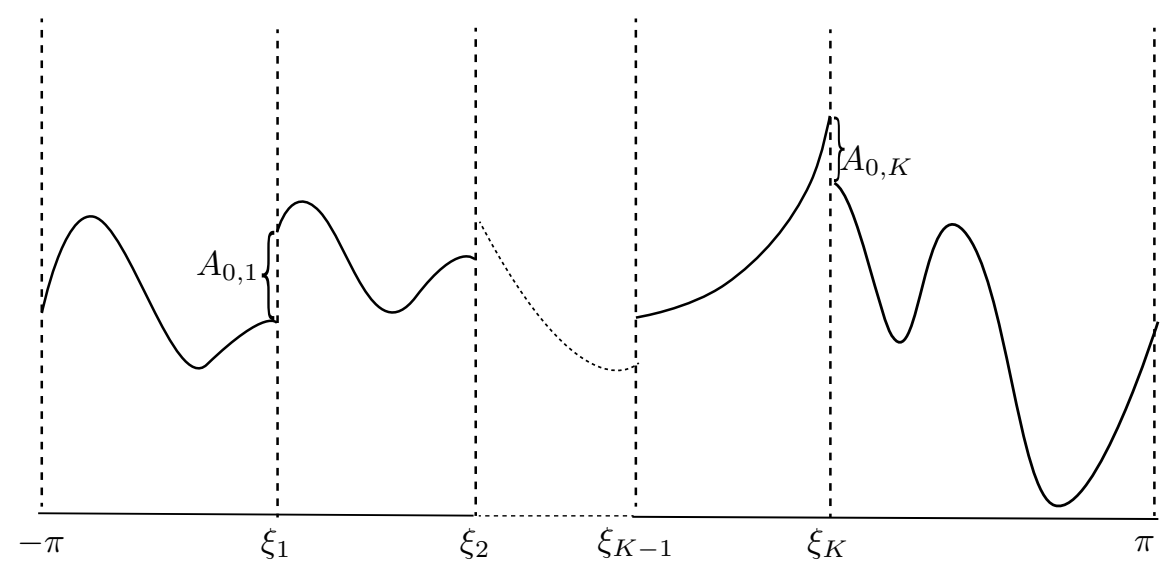

Figure 2.1. A piecewise smooth function

\section{The Algebraic ReCOnstruction Method}

Let us assume that $f$ has $K>0$ jump discontinuities $\left\{\xi_{j}\right\}_{j=1}^{K}$ (they can be located also at $\pm \pi$, but not necessarily so). Furthermore, we assume that $f \in C^{d}$ in every segment $\left(\xi_{j-1}, \xi_{j}\right)$, and we denote the associated jump magnitudes at $\xi_{j}$ by

$$
A_{l, j} \stackrel{\text { def }}{=} f^{(l)}\left(\xi_{j}^{+}\right)-f^{(l)}\left(\xi_{j}^{-}\right) .
$$

We write the piecewise smooth $f$ as the sum $f=\Psi+\Phi$, where $\Psi(x)$ is smooth and periodic and $\Phi(x)$ is a piecewise polynomial of degree $d$, uniquely determined by $\left\{\xi_{j}\right\},\left\{A_{i, j}\right\}$ such that it "absorbs" all the discontinuities of $f$ and its first $d$ derivatives. This idea is very old and goes back at least to A.N. Krylov (4, 28]). Eckhoff derives the following explicit representation for $\Phi(x)$ :

$$
\begin{aligned}
\Phi(x) & =\sum_{j=1}^{K} \sum_{l=0}^{d} A_{l, j} V_{l}\left(x ; \xi_{j}\right), \\
V_{n}\left(x ; \xi_{j}\right) & =-\frac{(2 \pi)^{n}}{(n+1) !} B_{n+1}\left(\frac{x-\xi_{j}}{2 \pi}\right) \quad \xi_{j} \leq x \leq \xi_{j}+2 \pi,
\end{aligned}
$$

where $V_{n}\left(x ; \xi_{j}\right)$ is understood to be periodically extended to $[-\pi, \pi]$ and $B_{n}(x)$ is the $n$-th Bernoulli polynomial. For completeness, let us derive the formula for the Fourier coefficients of $\Phi(x)$ (it can also be found in [15]).

Lemma 2.1. Let $\Phi(x)$ be a piecewise polynomial of degree d, with jump discontinuities $\left\{\xi_{j}\right\}_{j=1}^{K}$ and the associated jump magnitudes $\left\{A_{l, j}\right\}_{l=0, \ldots, d}^{j=1, \ldots, K}$. For definiteness, let us assume that $c_{0}(\Phi)=\int_{-\pi}^{\pi} \Phi(x) \mathrm{d} x=0$. Then

$$
c_{k}(\Phi)=\frac{1}{2 \pi} \sum_{j=1}^{K} \mathrm{e}^{-\imath k \xi_{j}} \sum_{l=0}^{d}(\imath k)^{-l-1} A_{l, j} .
$$

Proof. Denote $\Phi_{j}(x) \stackrel{\text { def }}{=} \sum_{l=0}^{d} A_{l, j} V_{l}\left(x ; \xi_{j}\right)$. These are periodic functions in $[-\pi, \pi]$ having a single discontinuity at $x=\xi_{j}$ and jump magnitudes $\left\{A_{0, j}, \ldots, A_{d, j}\right\}$. One 
integration by parts yields for $k \neq 0$,

$$
\begin{aligned}
c_{k}(\Phi) & =\frac{1}{2 \pi} \int_{-\pi}^{\pi} \mathrm{e}^{-\imath k x}\left(\sum_{j=0}^{K} \Phi_{j}(x)\right) \mathrm{d} x \\
& =\frac{1}{2 \pi \imath k} \sum_{j=0}^{K} A_{0, j} \mathrm{e}^{-\imath k \xi_{j}}+\frac{1}{\imath k} c_{k}\left(\sum_{j=0}^{K} \Phi_{j}^{\prime}\right),
\end{aligned}
$$

and so after $d+1$-fold repetition we obtain (using $\Phi_{j}^{(d+1)} \equiv 0$ ):

$$
c_{k}(\Phi)=\frac{1}{2 \pi} \sum_{l=0}^{d} \sum_{j=0}^{K}(\imath k)^{-l-1} A_{l, j} \mathrm{e}^{-\imath k \xi_{j}} .
$$

A key observation is that if $\Psi$ is sufficiently smooth, then the contribution of $c_{k}(\Psi)$ to $c_{k}(f)$ is negligible for large $k$. Therefore, for some large enough $M$ one can build from the equations (2.2) an approximate system:

$$
c_{k}(f) \approx \frac{1}{2 \pi} \sum_{j=1}^{K} \omega_{j}^{k} \sum_{l=0}^{d} \frac{A_{l, j}}{(\imath k)^{l+1}} \quad k=M, \ldots, M+d+1 .
$$

Here and in the rest of the paper we use the notation $\omega_{j} \stackrel{\text { def }}{=} \mathrm{e}^{-\imath \xi_{j}}$.

In fact, this system (up to a change of variables and the number of equations) lies at the heart of the algebraic reconstruction methods of Eckhoff [15, Banerjee and Geer [3], and Kvernadze [29. Banerjee and Geer solve it for all the parameters at once by least squares minimization. Eckhoff and Kvernadze eliminate all the $\left\{A_{i, j}\right\}$ first, resulting in a system of polynomial equations for the $\left\{\xi_{j}\right\}$, whose coefficients have nonlinear dependence on the initial data.

In contrast, we propose to solve this system separately for each $\xi=\xi_{j}$, beacuse this case reduces to a single polynomial equation with respect to $\xi$. We achieve this "separation" by filtering the original Fourier coefficients such that only the part related to a particular $\xi_{j}$ remains. This step requires some a priori knowledge of the approximate locations of the jumps. Fortunately, such information can easily be obtained by a variety of methods; see Section 5 .

Let us finish this section by presenting the main steps of the reconstruction. We denote the approximately reconstructed parameters with a tilde sign. If not stated otherwise, it is understood that these approximations depend on the index $M$. It is important to note that we distinguish between the actual smoothness of the function $f$ and the reconstruction order.

Algorithm 2.2. Let $f$ be a piecewise smooth function with jumps at $\left\{\xi_{j}\right\}_{j=1}^{K}$, continuously differentiable $d_{1}$ times between the jumps. Fix a reconstruction order to be some nonnegative integer $d \leq d_{1}$. Let there be given the first $M+d+2$ Fourier coefficients of $f$.

(1) Solve the system (3.1) by localization (Algorithm 5.1) and resolution (Algorithm (3.2). This will give us approximate values for the parameters $\left\{\widetilde{\xi}_{j}\right\}$ and $\left\{\widetilde{A}_{l, j}\right\}$. 
(2) Calculate the sequence

$$
c_{k}(\widetilde{\Phi})=\frac{1}{2 \pi} \sum_{j=1}^{K} \widetilde{\omega}_{j}^{k} \sum_{l=0}^{d} \frac{\widetilde{A}_{l, j}}{(\imath k)^{l+1}} \quad|k| \leq M
$$

and subsequently recover the approximate Fourier coefficients of the smooth part:

$$
c_{k}(\widetilde{\Psi}) \stackrel{\text { def }}{=} c_{k}(f)-c_{k}(\widetilde{\Phi}) \quad|k| \leq M .
$$

Take the final approximation to be

$$
\widetilde{f}=\widetilde{\Psi}+\widetilde{\Phi}=\sum_{|k| \leq M} c_{k}(\widetilde{\Psi}) \mathrm{e}^{\imath k x}+\sum_{j=1}^{K} \sum_{l=0}^{d} \widetilde{A}_{l, j} V_{l}\left(x ; \widetilde{\xi}_{j}\right) .
$$

The rest of this paper is devoted to providing all the details of the above algorithm and analyzing its accuracy. In particular, we shall prove that whenever $d_{1} \geq 2 d+1$, the following estimates hold:

$$
\begin{aligned}
\left|\widetilde{\xi}_{j}-\xi_{j}\right| & \leq C^{*}(d, K) \cdot F_{1}(\mathbb{A}, R, \mathbb{G}) \cdot M^{-d-2}, \\
\left|\widetilde{A}_{l, j}-A_{l, j}\right| & \leq C^{* *}(d, K) \cdot F_{2}(\mathbb{A}, R, \mathbb{G}) \cdot M^{l-d-1}, \\
|\widetilde{f}(x)-f(x)| & \leq C^{* * *}(d, K) \cdot F_{3}(\mathbb{A}, R, \mathbb{G}) \cdot M^{-d-1},
\end{aligned}
$$

where

- $C^{*}, C^{* *}, C^{* * *}$ are some absolute constants depending only on the "size" of the problem;

- $\mathbb{G}=\mathbb{G}\left(\xi_{1}, \ldots, \xi_{K}\right)$ represents the geometry of the jump points (such as minimal distance between two adjacent jumps);

- $\mathbb{A}=\mathbb{A}\left(\left|A_{0,1}\right|, \ldots,\left|A_{d_{1}, K}\right|\right)$ represents some a priori bounds on the jump magnitudes, such as lower and upper bounds;

- $R$ is an absolute bound for the Fourier coefficients of the smooth component $\Psi$

$$
\left|c_{k}(\Psi)\right| \leq R \cdot k^{-d-2}
$$

- $F_{1}, F_{2}, F_{3}$ are some "simple" functions.

In the course of our investigation we shall be defining more specific bounds, but it will always be assumed that those can be expressed in terms of the above quantities.

Since we are interested in "asymptotic" estimates, we will in general allow the inequalities (2.4) to hold for all $M$ starting from some index $K^{*}$ which may be large and depend on the parameters of the problem. However, if a particular bound holds for all $k>K^{*}$, then it will in general hold for $k=1,2, \ldots, K^{*}$ as well, with some larger multiplicative constants $\widetilde{C^{*}} \ldots$, but which are harder to compute explicitly.

Finally, let us mention that the reconstruction method described above is also applicable to situations where the function $f$ has different order of smoothness in each interval. In this case, parameters of each jump can be "resolved" up to its own corresponding order of accuracy (say $e_{j}$ ), while the whole function will be reconstructed up to the order $\sim M^{-\min _{j}}\left\{e_{j}\right\}-1$. 


\section{RESOLVING A SINGLE JUMP}

Let $K=1$, so that $f$ has a single jump $\xi$ and $\Phi=\sum_{l=0}^{d} A_{l} V_{l}(x ; \xi)$. Denote $\omega \stackrel{\text { def }}{=} \mathrm{e}^{-\imath \xi}$. The goal is to recover $\Phi$ from the approximate system of equations

$$
c_{k}(f) \approx \frac{\omega^{k}}{2 \pi} \sum_{l=0}^{d} \frac{A_{l}}{(\imath k)^{l+1}}\left(=c_{k}(\Phi)\right) \quad k=M, \ldots, M+d+1 .
$$

To find $\omega$, we eliminate $\left\{A_{0}, \ldots, A_{d}\right\}$ from the above equations. The result is a single polynomial equation having the exact value $\omega$ as one of its solutions. In Eckhoff's paper [14, this elimination is described in great detail, while here we present only the end result.

Let

$$
\begin{gathered}
m_{k} \stackrel{\text { def }}{=} 2 \pi(\imath k)^{d+1} c_{k}(\Phi)=\omega^{k} \sum_{l=0}^{d}(\imath k)^{d-l} A_{l}, \\
p_{k}^{d}(z) \stackrel{\text { def }}{=} \sum_{j=0}^{d+1}(-1)^{j}\left(\begin{array}{c}
d+1 \\
j
\end{array}\right) m_{k+j} z^{d+1-j} .
\end{gathered}
$$

Lemma 3.1. The point $\omega$ satisfies

$$
p_{k}^{d}(\omega)=0 \quad \forall k \in \mathbb{N} .
$$

Proof. This is an immediate consequence of Lemma A.4 (see Appendix A).

Since the exact coefficients $m_{k}$ (and as a result the polynomials $p_{k}^{d}(z)$ ) are unknown, we approximate these with the known quantities,

$$
\begin{aligned}
r_{k} & \stackrel{\text { def }}{=} 2 \pi(\imath k)^{d+1} c_{k}(f), \\
q_{k}^{d}(z) & \stackrel{\text { def }}{=} \sum_{j=0}^{d+1}(-1)^{j}\left(\begin{array}{c}
d+1 \\
j
\end{array}\right) r_{k+j} z^{d+1-j} .
\end{aligned}
$$

Now we are ready to formulate the procedure of recovering the parameters of a single jump.

Algorithm 3.2. Let us be given the first $M+d+2$ Fourier coefficients of the function $f$ which has a single jump ${ }^{1} \xi \in[-\pi, \pi]$.

(1) Solve the polynomial equation

$$
q_{M}^{d}(z)=0
$$

and take $\widetilde{\omega}$ to be the root which is closest to the unit circle. In Section 4 below, we shall provide the justification for this choice.

(2) The jump magnitudes $A_{0}, \ldots, A_{d}$ are reconstructed as follows. By (3.2), the exact values of $A_{j}$ satisfy

$$
m_{k} \omega^{-k}=\sum_{l=0}^{d}(\imath k)^{d-l} A_{l} \quad \forall k \in \mathbb{N} .
$$

\footnotetext{
${ }^{1}$ With the understanding that the points $\pm \pi$ are identified with each other.
} 
We use the approximations $r_{k} \approx m_{k}, \widetilde{\omega} \approx \omega$ and solve the system of linear equations

$$
r_{k} \widetilde{\omega}^{-k}=\sum_{l=0}^{d}(\imath k)^{d-l} \widetilde{A_{l}} \quad k=M, \ldots, M+d
$$

with respect to the unknowns $\left\{\widetilde{A}_{l}\right\}$ by any one of the standard methods.

\section{ACCURACY ANALYSIS: A SINGLE JUMP}

Our goal in this section is to analyze Algorithm 3.2 and calculate its accuracy. We shall express all our estimates in terms of the index $k$, keeping in mind that it should be replaced with $M$ to be consistent with the definitions of the previous sections.

4.1. Accuracy analysis: jump location. We start with the determination of the jump point $\widetilde{\omega}$. Our strategy will be to investigate the polynomial $q_{k}^{d}(z)$, and determine the bounds on locations of its roots. We can informally summarize the main results as follows.

(1) Starting from some $k$, the roots of $q_{k}^{d}(z)$ are "separated" from each other by at least $\sim k^{-1}$.

(2) If the function $f$ is continuously differentiable at least $d_{1} \geq 2 d+1$ times everywhere except at $\xi$, then one of those roots deviates from the "true" value $\omega$ by at most $\sim k^{-d-2}$.

We regard $q_{k}^{d}(z)$ as a perturbation of $p_{k}^{d}(z)$. With this point of view, we shall first describe the roots of $p_{k}^{d}(z)$, and then calculate the "deviations" due to the difference

$$
e_{k}^{d}(z)=q_{k}^{d}(z)-p_{k}^{d}(z)
$$

In the subsequent analysis we denote the roots of $p_{k}^{d}(z)$ by $z_{i}^{(k, d)}$ for $i=0,1, \ldots, d$, with the convention that $z_{0}^{(k, d)}=\omega$. Also, we denote the roots of $q_{k}^{d}(z)$ by $\kappa_{i}^{(k, d)}$.

It will be convenient to study $p_{k}^{d}(z)$ in a different coordinate system. For this purpose, consider the following transformation of the punctured $z$-plane:

$$
u=\mathcal{T}(z)=\frac{\omega}{z}-1 \quad z \neq 0 .
$$

Then the inverse map is given by

$$
z=\mathcal{T}^{-1}(u)=\frac{\omega}{u+1} \quad u \neq-1
$$

Now we translate the problem into the $u$-plane.

Definition 4.1. For all $k, d \in \mathbb{N}$ let

$$
s_{k}^{d}(u) \stackrel{\text { def }}{=} \frac{p_{k}^{d}(z)}{\omega^{k} z^{d+1}}=\frac{(u+1)^{d+1}}{\omega^{k+d+1}} p_{k}^{d}\left(\frac{\omega}{u+1}\right) .
$$

Claim 4.2. $s_{k}^{d}(u)$ is a polynomial function. Furthermore, if $u_{0} \neq-1$ is a root of $s_{k}^{d}(u)$, then $z_{0}=\mathcal{T}^{-1}\left(u_{0}\right)$ is a root of $p_{k}^{d}(u)$.

Therefore it makes sense to study the roots of $s_{k}^{d}(u)$. We denote these roots by $\sigma_{i}^{(k, d)}, i=0, \ldots, d$. The observation below is an immediate consequence of Lemma 3.1 . 
Claim 4.3. $s_{k}^{d}(0)=0$.

Therefore we will always take $\sigma_{0}^{(k, d)}=0$.

In what follows, we shall break $s_{k}^{d}(u)$ into a sum of terms and subsequently apply a perturbation analysis to determine its roots.

Lemma 4.4. The polynomial $s_{k}^{d}(u)$ can be written in the form $s_{k}^{d}(u)=\widetilde{s_{k}^{d}}(u)+h_{k}^{d}(u)$ where

$$
\begin{aligned}
\widetilde{s_{k}^{d}}(u) & \stackrel{\text { def }}{=} \sum_{s=1}^{d+1} a_{s-1, s} k^{s-1} u^{s}, \\
h_{k}^{d}(u) & \stackrel{\text { def }}{=} \sum_{s=2}^{d+1}\left(a_{0, s}+a_{1, s} k+\cdots+a_{s-2, s} k^{s-2}\right) u^{s},
\end{aligned}
$$

and also

$$
a_{s-1, s}=\left(\begin{array}{c}
d \\
s-1
\end{array}\right) \imath^{d} A_{0}(-1)^{d+1}(d+1-s) !\left(\begin{array}{c}
d+1 \\
s
\end{array}\right) .
$$

Proof. This is fairly straightforward but a somewhat tedious calculation, utilizing Lemma A.4 at some point. We leave this as an exercise.

Next we shall see that the dominant component $\widetilde{s_{k}^{d}}(u)$ determines the locations of the roots of $s_{k}^{d}(u)$ up to the first order accuracy, while the other component $h_{k}^{d}(u)$ is responsible for second order perturbations of these roots.

We denote the roots of $\widetilde{s}_{k}^{d}(u)$ by $\widetilde{\sigma}_{i}^{(k, d)}, i=0, \ldots, d$ with $\widetilde{\sigma}_{0}^{(k, d)}=0$.

It turns out that $\widetilde{s_{k}^{d}}(u)$ can be completely characterized.

Definition 4.5. For every $\alpha>-1$ and $n=0,1,2, \ldots$ let $\mathcal{L}_{n}^{(\alpha)}(x)$ denote the generalized Laguerre polynomial (11, Chapter 22], [0, Chapter 5.2]):

$$
\mathcal{L}_{n}^{(\alpha)}(x)=\sum_{m=0}^{n}\left(\begin{array}{c}
n+\alpha \\
n-m
\end{array}\right) \frac{(-x)^{m}}{m !} .
$$

Lemma 4.6. Assume the above notations. Then

(1) The polynomial $\widetilde{s_{k}^{d}}(u)$ satisfies

$$
\widetilde{s_{k}^{d}}(u)=\frac{1}{k} \widetilde{s_{1}^{d}}(k u) .
$$

(2) Furthermore,

$$
\widetilde{s_{1}^{d}}(u)=-(-\imath)^{d} A_{0}(d+1) ! \mathcal{L}_{d+1}^{(-1)}(-u) .
$$

Proof. Straightforward substituion of (4.4) into (4.3).

Corollary 4.7. For all $k \in \mathbb{N}, \widetilde{s}_{k}^{d}\left(u^{*}\right)=0$ if and only if $\mathcal{L}_{d+1}^{(-1)}\left(-k u^{*}\right)=0$.

Lemma 4.8. The numbers $\left\{\widetilde{\sigma}_{i}^{(k, d)}\right\}$ satisfy the following properties:

(1) each $\widetilde{\sigma}_{i}^{(k, d)}$ is a simple root of $\widetilde{s}_{k}^{d}(u)$;

(2) $\widetilde{\sigma}_{i}^{(k, d)}<0$ for $i=1,2, \ldots, d$;

(3) there exist constants $C_{1}, C_{2}$ such that for every $k \in \mathbb{N}$ and $0 \leq i<j \leq d$,

$$
C_{1} k^{-1} \leq\left|\widetilde{\sigma}_{i}^{(k, d)}-\widetilde{\sigma}_{j}^{(k, d)}\right| \leq C_{2} k^{-1} .
$$


Proof. Following Corollary 4.7, we only need to characterize the roots of $\mathcal{L}_{d+1}^{(-1)}(-u)$. By [40, Chapter 5.2], for every integer $m \geq 1$,

$$
\mathcal{L}_{n}^{(-m)}(x)=(-x)^{m} \frac{(n-m) !}{n !} \mathcal{L}_{n-k}^{(m)}(x),
$$

and therefore

$$
\mathcal{L}_{d+1}^{(-1)}(-u)=u \frac{d !}{(d+1) !} \mathcal{L}_{d}^{(1)}(-u) .
$$

The polynomials $\left\{\mathcal{L}_{n}^{(1)}(x)\right\}_{n=0}^{\infty}$ form an orthogonal system on the interval $(0, \infty)$ (see again [1, Chapter 22], 40, Chapter 5.2]). Parts (1) and (2) follow immediately. Part (3) follows by taking $C_{1}$ and $C_{2}$ to be the minimal and the maximal distance between the roots of $\mathcal{L}_{d}^{(1)}(u)$, correspondingly.

Now we show that $h_{k}^{d}(u)$ perturbs the zeros of $\widetilde{s_{k}^{d}}(u)$ by at most $\sim k^{-2}$. Since the coefficients $h_{k}^{d}(u)$ depend linearly on $A_{0}, \ldots, A_{d}$, we can expect that the bound will depend on the quantity $\sum_{l=0}^{d}\left|A_{l}\right|$. For convenience, let us therefore define

$$
A^{*} \stackrel{\text { def }}{=} \max \left(1, \sum_{l=0}^{d}\left|A_{l}\right|\right) \text {. }
$$

Lemma 4.9. There exist constants $C_{3}, K_{1}$ such that for all $k>K_{1} A^{*}$ and for all $i=0, \ldots, d$,

$$
\left|\widetilde{\sigma}_{i}^{(k, d)}-\sigma_{i}^{(k, d)}\right| \leq C_{3} A^{*} k^{-2} .
$$

Proof. Our method of proof is based on Rouche's theorem (Theorem A.5). We shall define a sequence $\rho(k)=C_{3} A^{*} k^{-2}$ (where $C_{3}$ is to be determined) and consider disks of radius $\rho(k)$ around each one of the roots $\widetilde{\sigma}_{i}^{(k, d)}$. Our goal is to find $C_{3}$ so that $\left|\widetilde{s_{k}^{d}}\left(u_{\theta}\right)\right|>\left|h_{k}^{d}\left(u_{\theta}\right)\right|$ for all points $u_{\theta}=\widetilde{\sigma}_{i}^{(k, d)}+\rho(k) \mathrm{e}^{\imath \theta}$ on the boundaries of these disks.

- In order to bound $\left|\tilde{s}_{k}^{d}\left(u_{\theta}\right)\right|$ from below, we shall use Lemma A.6. We need to bound from below the first derivative at $\widetilde{\sigma}_{i}^{(k, d)}$, as well as to bound from above the second derivative in the disk $B_{k^{-1}}\left(\tilde{\sigma}_{i}^{(k, d)}\right)$.

(1) We always have

$\left.\frac{\mathrm{d}}{\mathrm{d} u} \tilde{s}_{k}^{d}(u)\right|_{u=\widetilde{\sigma}_{i}^{(k, d)}}=\left.\frac{\mathrm{d}}{\mathrm{d} u}\left(\frac{1}{k} \widetilde{s}_{1}^{d}(k u)\right)\right|_{u=\widetilde{\sigma}_{i}^{(k, d)}}=\left.\frac{\mathrm{d} \widetilde{s}_{1}^{d}(w)}{\mathrm{d} w}\right|_{w=k \widetilde{\sigma}_{i}^{(k, d)}}$.

Now $k \widetilde{\sigma}_{i}^{(k, d)}$ is always a root of $\widetilde{s}_{1}^{d}(u)$, therefore the value of

$$
\left.\frac{\mathrm{d}}{\mathrm{d} u} \tilde{s}_{k}^{d}(u)\right|_{u=\tilde{\sigma}_{i}^{(k, d)}}
$$

is independent of $k$ and thus we can write

$$
\left.\left|\frac{\mathrm{d}}{\mathrm{d} u} \widetilde{s}_{k}^{d}(u)\right|_{u=\widetilde{\sigma}_{i}^{(k, d)}}\left|\geq C_{4} \stackrel{\text { def }}{=} \min _{i}\right| \frac{\mathrm{d}}{\mathrm{d} u} \widetilde{s}_{1}^{d}(u)\right|_{u=\widetilde{\sigma}_{i}^{(1, d)}} \mid .
$$

Since all the roots are simple, this is guaranteed to be a strictly positive bound. 
(2) Now consider a point $u^{*} \in B_{k^{-1}}\left(\widetilde{\sigma}_{i}^{(k, d)}\right)$. Then $\left|k u^{*}-k \widetilde{\sigma}_{i}^{(k, d)}\right| \leq 1$ and therefore $k u^{*} \in B_{1}\left(\widetilde{\sigma}_{i}^{(1, d)}\right)$. Using (4.5) and differentiating twice, we get

$$
\left.\frac{\mathrm{d}^{2}}{\mathrm{~d} u^{2}} \widetilde{s}_{k}^{d}(u)\right|_{u=u^{*}}=\left.k \frac{\mathrm{d}^{2}}{\mathrm{~d} w^{2}} \widetilde{s}_{1}^{d}(w)\right|_{w=k u^{*}} .
$$

Let

$$
C_{5} \stackrel{\text { def }}{=} \max _{i} \max _{w^{*} \in B_{1}\left(\widetilde{\sigma}_{i}^{(1, d)}\right)}\left|\frac{\mathrm{d}^{2}}{\mathrm{~d} w^{2}} \widetilde{s}_{1}^{d}(w)\right|_{w=w^{*}} \mid .
$$

(3) The constants $C_{4}$ and $C_{5}$ therefore satisfy the assumptions of Lemma A.6. We define $C_{6} \stackrel{\text { def }}{=} \min \left(1, \frac{C_{4}}{C_{5}}\right)$. The conclusion is that there exists a constant $C_{7}$ such that for every function $\eta(k): \mathbb{N} \rightarrow \mathbb{R}$ satisfying $0<\eta(k)<\frac{C_{6}}{k}$ we have

$$
\left|\widetilde{s}_{k}^{d}\left(\tilde{\sigma}_{i}^{(k, d)}+\eta(k) \mathrm{e}^{\imath \theta}\right)\right|>C_{7} \eta(k) .
$$

- Now we shall bound $\left|h_{k}^{d}\left(u_{\theta}\right)\right|$ from above. Recall that

$$
h_{k}^{d}(u)=\sum_{s=2}^{d+1}\left(a_{0, s}+a_{1, s} k+\cdots+a_{s-2, s} k^{s-2}\right) u^{s}
$$

where $a_{i, j}$ are some linear functions of $A_{0}, \ldots, A_{d}$. Let $\zeta(k): \mathbb{N} \rightarrow \mathbb{R}$ be any function satisfying $0<\zeta(k)<\frac{1}{k}$, and consider $u_{\theta}=\widetilde{\sigma}_{i}^{(k, d)}+\zeta(k) \mathrm{e}^{\imath \theta}$. By Lemma 4.8. $\left|\widetilde{\sigma}_{i}^{(k, d)}\right|<C_{2} k^{-1}$ and therefore $\left|u_{\theta}\right|<2 \cdot \max \left(1, C_{2}\right) k^{-1}$. But then

$$
\begin{aligned}
\left|h_{k}^{d}\left(u_{\theta}\right)\right| \leq & \left|a_{0,2}\right|\left|u_{\theta}\right|^{2}+\left(\left|a_{0,3}\right|+\left|a_{1,3}\right| k\right)\left|u_{\theta}\right|^{3}+\ldots \\
& +\left(\left|a_{0, d+1}\right|+\cdots+\left|a_{d-1, d+1}\right| k^{d-1}\right)\left|u_{\theta}\right|^{d+1} \leq C_{8} A^{*} k^{-2}
\end{aligned}
$$

for some constant $C_{8}$.

Set $C_{3} \stackrel{\text { def }}{=} \frac{2 C_{8}}{C_{7}}$ and let $\rho(k)=C_{3} A^{*} k^{-2}$. Let $k>\underbrace{\frac{2 C_{8}}{C_{7} C_{6}}}_{\stackrel{\text { def }}{=} K_{1}} A^{*}$. Then $\rho(k)<\frac{C_{6}}{k}$ and therefore

$$
\left|\tilde{s}_{k}^{d}\left(\tilde{\sigma}_{i}^{(k, d)}+\rho(k) \mathrm{e}^{\imath \theta}\right)\right|>\underbrace{C_{7} \rho(k)}_{=2 C_{8} A^{*} k^{-2}}>C_{8} A^{*} k^{-2} \geq\left|h_{k}^{d}\left(\tilde{\sigma}_{i}^{(k, d)}+\rho(k) \mathrm{e}^{\imath \theta}\right)\right|,
$$

which completes the proof.

Remark 4.10. We have in fact shown that for each $k>K_{1}$ the polynomial $s_{k}^{d}(u)$ has precisely $d+1$ distinct roots.

Now we can go back to the original polynomial $p_{k}^{d}(z)$ and accurately describe the location of its roots $\left\{z_{i}^{(k, d)}\right\}$. Recall from Claim4.2 that $z_{i}^{(k, d)}=\mathcal{T}^{-1}\left(\sigma_{i}^{(k, d)}\right)$. Being careful to avoid the singularity $\sigma_{i}^{(k, d)}=-1$ (by choosing large enough $k$ ), we now show that the geometry of the roots $\sigma_{i}^{(k, d)}$ is preserved under $\mathcal{T}^{-1}$. In 
particular, the numbers $z_{i}^{(k, d)}$ remain separated from each other (following (4.6)), each of them being close (following Lemma 4.9) to one of the numbers

$$
y_{i}^{(k, d)} \stackrel{\text { def }}{=} \mathcal{T}^{-1}\left(\widetilde{\sigma}_{i}^{(k, d)}\right)=\frac{\omega}{\widetilde{\sigma}_{i}^{(k, d)}+1} .
$$

The only thing which is different are the constants.

Lemma 4.11. Let $y_{i}^{(k, d)}$ be defined by (4.8). Then:

(1) there exist constants $C_{9}, C_{10}, K_{2}$ such that for all $k>K_{2}$ and $0 \leq i<j \leq d$,

$$
C_{9} k^{-1} \leq\left|y_{i}^{(k, d)}-y_{j}^{(k, d)}\right| \leq C_{10} k^{-1}
$$

(2) there exist constants $C_{11}, K_{3}$ such that for all $k>K_{3} A^{*}$,

$$
\left|z_{i}^{(k, d)}-y_{i}^{(k, d)}\right|<C_{11} A^{*} \cdot k^{-2}
$$

(3) there exist constants $C_{12}, C_{13}, K_{4}$ such that for all $k>K_{4} A^{*}$ and $0 \leq i<$ $j \leq d$,

$$
C_{12} k^{-1} \leq\left|z_{i}^{(k, d)}-z_{j}^{(k, d)}\right| \leq C_{13} k^{-1}
$$

Proof. If $k>2 C_{2}$, then $\left|\widetilde{\sigma}_{i}^{(k, d)}\right|<\frac{1}{2}$ (see (4.6) $)$. It follows that $\frac{1}{2}<\left|\widetilde{\sigma}_{i}^{(k, d)}+1\right| \leq 1$ and so by (4.8),

$$
C_{1} k^{-1}<\left|y_{i}^{(k, d)}-y_{j}^{(k, d)}\right|<4 C_{2} k^{-1}
$$

This proves (11) with $C_{9}=C_{1}, C_{10}=4 C_{2}$ and $K_{2}=2 C_{2}$.

If, in addition, $k>\frac{2 C_{3}}{C_{1}}$, then $\left|\widetilde{\sigma}_{i}^{(k, d)}-\sigma_{i}^{(k, d)}\right|<\frac{\left|\widetilde{\sigma}_{i}^{(k, d)}\right|}{2}<\frac{1}{4}$ and therefore $\left|\sigma_{i}^{(k, d)}+1\right|>\frac{1}{4}$. It follows from (4.7) that

$$
\left|z_{i}^{(k, d)}-y_{i}^{(k, d)}\right|=\frac{\left|\widetilde{\sigma}_{i}^{(k, d)}-\sigma_{i}^{(k, d)}\right|}{\left|\widetilde{\sigma}_{i}^{(k, d)}+1\right|\left|\sigma_{i}^{(k, d)}+1\right|}<4 C_{3} k^{-2} \quad k>\underbrace{\max \left(2 C_{2}, \frac{2 C_{3}}{C_{1}}, K_{1}\right)}_{\stackrel{\text { def }}{=} K_{3}} A^{*}
$$

and this proves (2) with $C_{11}=4 C_{3}$ and $K_{3}$ as above.

Let $k>\max \left(K_{2}, K_{3}, \frac{4 C_{11}}{C_{9}}\right) A^{*}$. Using (1) and (2), we have on one hand,

$$
\begin{aligned}
z_{i}^{(k, d)}-z_{j}^{(k, d)} \mid & \stackrel{\text { def }}{=} K_{4} \\
& <\left|y_{i}^{(k, d)}-y_{j}^{(k, d)}\right|+\left|z_{i}^{(k, d)}-y_{i}^{(k, d)}\right|+\left|y_{j}^{(k, d)}-y_{j}^{(k, d)}\right|+\frac{C_{11}}{k^{2}}+\frac{C_{11}}{k^{2}} \\
& <\left|y_{i}^{(k, d)}-y_{j}^{(k, d)}\right|+2 \cdot \frac{C_{9}}{4 k}<\frac{3}{2}\left|y_{i}^{(k, d)}-y_{j}^{(k, d)}\right|<\underbrace{\frac{3}{2} C_{10}}_{\stackrel{\text { def }}{=} C_{13}} k^{-1},
\end{aligned}
$$


and on the other hand,

$$
\begin{aligned}
\left|z_{i}^{(k, d)}-z_{j}^{(k, d)}\right| & >\left|y_{i}^{(k, d)}-y_{j}^{(k, d)}\right|-\left|y_{j}^{(k, d)}-z_{j}^{(k, d)}\right|-\left|y_{j}^{(k, d)}-z_{j}^{(k, d)}\right| \\
& >\frac{1}{2}\left|y_{i}^{(k, d)}-y_{j}^{(k, d)}\right|>\underbrace{\frac{1}{2} C_{9}}_{\stackrel{\text { def }}{=} C_{12}} k^{-1} .
\end{aligned}
$$

That proves (3).

Remaining in the $z$-plane, we now turn to investigate $q_{k}^{d}(z)$ and its roots $\left\{\kappa_{i}^{(k, d)}\right\}$. Recall that we consider $q_{k}^{d}(z)$ to be a "perturbation" of $p_{k}^{d}(z)$ by another polynomial $e_{k}^{d}(z)($ see $(3.2)$ and $(3.3))$ :

$$
q_{k}^{d}(z)=p_{k}^{d}(z)+e_{k}^{d}(z) .
$$

The coefficients of $e_{k}^{d}(z)$ depend on the Fourier coefficients of the "smooth part" of our pieceiwise smooth function $f$. It turns out that in the general setting, the coefficients of $e_{k}^{d}(z)$ are large compared to those of $p_{k}^{d}(z)$ and therefore the perturbations of the roots are large too. If, however, there is enough structure in those coefficients due to additional orders of smoothness, then the perturbation of the roots is small. This is the essence of the key Lemma 4.12 below.

Recall that $f$ has in fact $d_{1} \geq d$ continuous derivatives everywhere in $[-\pi, \pi] \backslash\{\xi\}$, and denote the additional jump magnitudes at $\xi$ by $A_{d+1}, \ldots, A_{d_{1}}$. For every $l \leq d_{1}$, let $\Phi_{l}$ denote the piecewise polynomial of degree $l$ with jump point $\xi$ and jump magnitudes $A_{0}, \ldots, A_{l}$. Then we write

$$
f=\Phi_{d}+\left(\Phi_{d_{1}}-\Phi_{d}\right)+\Psi^{*}
$$

where $\Psi^{*}$ is $d_{1}$-times smooth everywhere in $[-\pi, \pi]$. Thus there exists a constant $R^{*}$ such that

$$
\left|c_{k}\left(\Psi^{*}\right)\right| \leq R^{*} k^{-d_{1}-2} .
$$

Let us also denote

$$
\begin{aligned}
& A^{* *} \stackrel{\text { def }}{=} \max \left(1, \sum_{l=d+1}^{d_{1}}\left|A_{l}\right|\right), \\
& H \stackrel{\text { def }}{=}\left(A^{*}+A^{* *}+R^{*}\right) .
\end{aligned}
$$

Lemma 4.12. Let $d_{1} \geq 2 d+1$. Then there exist constants $C_{14}, C_{15}, K_{5}$ such that for all $k>K_{5} H$,

$$
\left|\kappa_{i}^{(k, d)}-z_{i}^{(k, d)}\right| \leq \begin{cases}C_{14} \cdot H \cdot k^{-2} & i=1,2, \ldots, d, \\ C_{15} \cdot H \cdot k^{-d-2} & i=0 .\end{cases}
$$

Proof. The idea of the proof is the same as in Lemma 4.9. Namely, we shall seek the constants $C_{14}, C_{15}$ and $K_{5}$ such that if $\rho_{1}(k)=C_{14} \cdot H \cdot k^{-2}$ and $\rho_{2}(k)=$ $C_{15} \cdot H \cdot k^{-d-2}$, then for $i=0,1, \ldots, d$ and $k>K_{5} H$ there exist neighborhoods $D_{i}^{(k)}$ of $z_{i}^{(k, d)}$ such that $\left|p_{k}^{d}(z)\right|>\left|e_{k}^{d}(z)\right|$ on the boundary of $D_{i}^{(k)}$ and

- $\operatorname{diam} D_{i}^{(k)}=\rho_{1}(k)$ for $i=1,2, \ldots, d$;

- $\operatorname{diam} D_{0}^{(k)}=\rho_{2}(k)$. 
(1) In order to show that $\left|p_{k}^{d}(z)\right|$ is large in some neighborhood of $z_{i}^{(k, d)}$, let us first show that $\left|s_{k}^{d}(u)\right|$ is large in some neighborhood of $\sigma_{i}^{(k, d)}$. Recall that $s_{k}^{d}(u)=\widetilde{s}_{k}^{d}(u)+h_{k}^{d}(u)$. We have shown in the proof of Lemma4.9 that if $\eta(k)$ is any function satisfying $0<\eta(k)<\frac{C_{6}}{k}$, then $\left|\widetilde{s}_{k}^{d}(u)\right|>C_{7} \eta(k)$ everywhere on $\partial B_{\eta(k)}\left(\widetilde{\sigma}_{i}^{(k, d)}\right)$. Furthermore, in this case $\left|h_{k}^{d}(u)\right|<C_{8} A^{*} k^{-2}$. We now require that

$$
C_{8} A^{*} k^{-2}<\frac{1}{2} C_{7} \eta(k)
$$

which is true if $k>\frac{2 C_{8} A^{*}}{C_{7} C_{6}}=K_{1} A^{*}$. In that case we have

$$
\left|s_{k}^{d}(u)\right|>\frac{1}{2} C_{7} \eta(k) \quad \forall u \in B_{\eta(k)}\left(\widetilde{\sigma}_{i}^{(k, d)}\right) .
$$

This is almost what we want - we would like to have such a bound on the boundary of a neighborhood of $\sigma_{i}^{(k, d)}$ instead of $\widetilde{\sigma}_{i}^{(k, d)}$. If $i=0$, then these values coincide, and so we are done. Otherwise, recall that for $k>K_{1} A^{*}$ we also have $\left|\widetilde{\sigma}_{i}^{(k, d)}-\sigma_{i}^{(k, d)}\right| \leq C_{3} A^{*} k^{-2}$. So in order to make sure that $\sigma_{i}^{(k, d)}$ belongs to $B_{\eta(k)}\left(\widetilde{\sigma}_{i}^{(k, d)}\right)$, we just require that $\eta(k) \geq C_{3} A^{*} k^{-2}$.

We have thus shown the following:

(a) For every function $0<\eta(k)<\frac{C_{6}}{k}$ and for every $k>K_{1} A^{*}$, the following bound holds for every $u$ on the boundary of a neighborhood of $\sigma_{0}^{(k, d)}=0$ of diameter $2 \eta(k)$ :

$$
\left|s_{k}^{d}(u)\right|>\frac{1}{2} C_{7} \eta(k)
$$

(b) For every $k>K_{1} A^{*}$ and $\eta(k)$ as above, which additionally satisfies $\eta(k) \geq C_{3} A^{*} k^{-2}$, the above bound holds for every $u$ on the boundary of a neighborhood of $\sigma_{i}^{(k, d)}$ of the same diameter $2 \eta(k)$, for every $i=$ $0,1, \ldots, d$.

(2) We can now show that similar bounds hold for $p_{k}^{d}(z)$. Again, only the constants will be different. The map $\mathcal{T}^{-1}$ (4.1), being a Möbius transformation, maps $B_{\eta(k)}\left(\widetilde{\sigma}_{i}^{(k, d)}\right)$ to a circular neighborhood of $z_{i}^{(k, d)}$ (which is not necessarily centered at $\left.z_{i}^{(k, d)}\right)$. Let $u^{*} \in B_{\eta(k)}\left(\tilde{\sigma}_{i}^{(k, d)}\right)$. Now $\left|u^{*}-\widetilde{\sigma}_{i}^{(k, d)}\right| \leq C_{6} k^{-1}$ and also $-\frac{C_{2}}{k}<\widetilde{\sigma}_{i}^{(k, d)}<0$. Therefore, if $k>$ $2\left(C_{2}+C_{6}\right)$, then $\Re\left(u^{*}\right)>-\frac{1}{2}$ and so $\left|u^{*}+1\right|>\frac{1}{2}$. On the other hand, in this case $\left|u^{*}+1\right|<2$.

Now let $u_{1}$ and $u_{2}$ be two points in the $u$-plane, such that $\left|u_{1}-u_{2}\right|=r$ and $\frac{1}{2}<\left|u_{1}\right|,\left|u_{2}\right|<2$. They are mapped to the $z$-plane such that

$$
\frac{r}{4}<\left|\frac{\omega}{u_{1}+1}-\frac{\omega}{u_{2}+1}\right|<4 r .
$$

Recalling (4.11) and (4.2), we conclude:

(a) For every function $0<\eta(k)<\frac{C_{6}}{k}$ and every

$$
k>\underbrace{\max \left(K_{1}, 2\left(C_{2}+C_{6}\right)\right)}_{\stackrel{\text { def }}{=} K_{6}} A^{*},
$$


there exists a circular neighborhood of $\omega$ having diameter between $\frac{\eta(k)}{2}$ and $8 \eta(k)$, such that the magnitude of $p_{k}^{d}(z)$ on the boundary of this neighborhood satisfies

$$
\left|p_{k}^{d}(z)\right|=\left|z^{d+1}\right|\left|s_{k}^{d}(u)\right|>2^{-d-2} C_{7} \eta(k)=C_{16} \eta(k) .
$$

(b) For every $k>K_{6} A^{*}$ and $\eta(k)$ as above, which additionally satisfies $\eta(k) \geq C_{3} A^{*} k^{-2}$, the above bound holds for every $z$ on the boundary of a neighborhood of $z_{i}^{(k, d)}$ of the same diameter as above, for every $i=0,1, \ldots, d$.

(3) Once we have the lower bound for $\left|p_{k}^{d}(z)\right|$ on circles of diameter at most $8 \eta(k)<\frac{8 C_{6}}{k}$ containing $z_{i}^{(k, d)}$, we can now establish an upper bound for $\left|e_{k}^{d}(z)\right|$ on these circles. Let $z_{*}$ belong to such a circle. On one hand, its distance from $z_{i}^{(k, d)}$ is at most $\frac{8 C_{6}}{k}$. On the other hand, by Lemma 4.11, $\left|z_{i}^{(k, d)}-\omega\right|<\frac{C_{13}}{k}$ for all $k>K_{4} A^{*}$. Therefore $\left|z_{*}-\omega\right|<\frac{8 C_{6}+C_{13}}{k}$. Denote $C_{17} \stackrel{\text { def }}{=} 8 C_{6}+C_{13}$ and let $z_{\theta}=\omega+\zeta(k) \mathrm{e}^{\imath \theta}$ where $\zeta(k)$ is some function satisfying $0<\zeta(k)<\frac{C_{17}}{k}$. Our goal now is to find a uniform upper bound for $\left|e_{k}^{d}\left(z_{\theta}\right)\right|$.

(a) By (4.9) we have

$$
\begin{aligned}
r_{k}-m_{k} & =2 \pi(\imath k)^{d+1}\left\{c_{k}(f)-c_{k}\left(\Phi_{d}\right)\right\} \\
& =\omega^{k} \cdot \sum_{l=1}^{d_{1}-d} \frac{A_{d+l}}{(\imath k)^{l}}+\underbrace{2 \pi(\imath k)^{d+1} c_{k}\left(\Psi^{*}\right)}_{\stackrel{\text { def }}{=} \delta_{k}}
\end{aligned}
$$

Therefore,

$$
\begin{aligned}
& e_{k}^{d}\left(z_{\theta}\right)=\sum_{j=0}^{d+1}(-1)^{j}\left(\begin{array}{c}
d+1 \\
j
\end{array}\right)\left\{\omega^{k+j} \cdot \sum_{l=1}^{d_{1}-d} \frac{A_{d+l}}{(\imath(k+j))^{l}}+\delta_{k+j}\right\} z_{\theta}^{d+1-j} \\
& =\underbrace{\sum_{l=1}^{d_{1}-d}(-\imath)^{l} A_{d+l} \sum_{j=0}^{d+1} \frac{(-1)^{j}}{(k+j)^{l}}\left(\begin{array}{c}
d+1 \\
j
\end{array}\right) \omega^{k+j} z_{\theta}^{d+1-j}}_{\stackrel{\text { def }}{=} \Lambda_{k}\left(z_{\theta}\right)} \\
& +\underbrace{\sum_{j=0}^{d+1}(-1)^{j}\left(\begin{array}{c}
d+1 \\
j
\end{array}\right) \delta_{k+j} z_{\theta}^{d+1-j}}_{\stackrel{\text { def }}{=} \Delta_{k}\left(z_{\theta}\right)} .
\end{aligned}
$$

(b) On one hand, we have the bound (4.10). On the other hand, $\left|z_{\theta}\right|<2$ and therefore,

$$
\left|\Delta_{k}\left(z_{\theta}\right)\right| \leq C_{18} 2^{d+1} \cdot 2 \pi \cdot k^{d+1}\left|c_{k}(\Psi)\right| \leq \frac{C_{19} R^{*}}{k^{d_{1}-d+1}}
$$

for some $C_{19}$.

(c) Now we need to estimate $\Lambda_{k}\left(z_{\theta}\right)$. First,

$$
\begin{aligned}
z_{\theta}^{d+1-j} & =\left(\omega+\zeta(k) \mathrm{e}^{\imath \theta}\right)^{d+1-j} \\
& =\omega^{d+1-j}+(d+1-j) \omega^{d-j} \zeta(k) \mathrm{e}^{\imath \theta}+\alpha_{j}(k)
\end{aligned}
$$


where $\left|\alpha_{j}(k)\right| \leq C_{20} \zeta^{2}(k)$ for some constant $C_{20}$. Furthermore, using the estimate of Lemma A.3 we have

$$
\begin{aligned}
\Lambda_{k}\left(z_{\theta}\right)= & \omega^{k+d+1} \sum_{l=1}^{d_{1}-d} \frac{A_{d+l}}{l^{l}} \underbrace{\sum_{j=0}^{d+1}(-1)^{j}\left(\begin{array}{c}
d+1 \\
j
\end{array}\right) \frac{1}{(k+j)^{l}}}_{|\cdot| \leq C_{21} \cdot k^{-d-l-1}} \\
& +\zeta(k) \mathrm{e}^{\imath \theta}(d+1) \omega^{k+d} \sum_{l=1}^{d_{1}-d} \frac{A_{d+l}}{\imath^{l}} \underbrace{\sum_{j=0}^{d=0}(-1)^{j}\left(\begin{array}{c}
d \\
j
\end{array}\right) \frac{1}{(k+j)^{l}}}_{|\cdot| \leq C_{22} \cdot k^{-d-l}} \\
& \underbrace{\sum_{l=1}^{d_{1}-d}(-\imath)^{l} A_{d+l} \sum_{j=0}^{d+1} \frac{(-1)^{j}}{(k+j)^{l}}\left(\begin{array}{c}
d+1 \\
j
\end{array}\right) \omega^{k+j} \alpha_{j}(k)}_{|\cdot| \leq C_{23} \cdot k^{-1} \zeta^{2}(k)}
\end{aligned}
$$

for all $k>K_{7}$ where $K_{7}$ is an explicit constant (see Lemma A.3). Therefore,

$$
\left|\Lambda_{k}\left(z_{\theta}\right)\right|<A^{* *} \times\left\{C_{24} \cdot k^{-d-2}+C_{25} \zeta(k) k^{-d-1}+C_{26} \cdot k^{-1} \zeta^{2}(k)\right\} .
$$

Combining all of the above estimates we therefore have for $k>$ $\max \left(K_{4}, K_{7}\right) A^{*}$,

$$
\left|e_{k}^{d}\left(z_{\theta}\right)\right|<A^{* *} \times\left(\frac{C_{24}}{k^{d+2}}+\frac{C_{25}}{k^{d+1}} \zeta(k)+\frac{C_{26}}{k} \zeta^{2}(k)\right)+\frac{C_{19} R^{*}}{k^{d_{1}-d+1}} .
$$

(4) We can finally compare $\left|p_{k}^{d}(z)\right|$ and $\left|e_{k}^{d}(z)\right|$. Let $k>\underbrace{\max \left(K_{4}, K_{7}, K_{6}\right)}_{\stackrel{\text { def }}{=} K_{8}} A^{*}$ and consider two cases.

(a) Suppose $z_{i}^{(k, d)} \neq \omega$. Set $\rho(k)=\frac{C_{14} H}{8 k^{2}}$ where $C_{14}$ is to be determined, and suppose also that

$$
C_{3} A^{*} k^{-2} \leq \rho(k)<\frac{C_{6}}{k}
$$

We have shown above that there exists a neighborhood $D_{i}^{(k)}$ containing $z_{i}^{(k, d)}$ of diameter at most $8 \rho(k)=C_{14} \cdot H \cdot k^{-2}$ such that for every $z^{*} \in \partial D_{i}^{(k, d)}$ we have $\left|p_{k}^{d}\left(z^{*}\right)\right|>C_{16} \rho(k)=\frac{C_{16} C_{14} H}{8 k^{2}}$. On the other hand, for every such $z^{*}$ we have by (4.13),

$$
\begin{aligned}
\left|e_{k}^{d}\left(z^{*}\right)\right| & <A^{* *} \times\left(\frac{C_{24}}{k^{d+2}}+\frac{C_{25}}{k^{d+1}} \rho(k)+\frac{C_{26}}{k} \rho^{2}(k)\right)+\frac{C_{19} R^{*}}{k^{d_{1}-d+1}} \\
& <\frac{A^{* *} \times\left(C_{24}+C_{25} C_{6}+C_{26} C_{6}^{2}\right)+C_{19} R^{*}}{k^{2}}<\left(A^{* *}+R^{*}\right) \frac{C_{27}}{k^{2}}
\end{aligned}
$$


Therefore we must choose $C_{14}$ and $k$ for which the condition $\frac{C_{16} C_{14} H}{8 k^{2}}>$ $\frac{C_{27}\left(A^{* *}+R^{*}\right)}{k^{2}}$ is satisfied, together with (4.14). For example:

$$
\begin{aligned}
& C_{14} \stackrel{\text { def }}{=} \max \left(\frac{8 C_{27}}{C_{16}}, 8 C_{3}\right), \\
& k>\underbrace{\max \left(K_{8}, \frac{C_{14}}{8 C_{6}}\right)}_{\stackrel{\text { def }}{=} K_{5}} \times H .
\end{aligned}
$$

In this case, $q_{k}^{d}(z)$ has a simple zero $\kappa_{i}^{(k, d)}$ in $D_{i}^{(k)}$ so that

$$
\left|\kappa_{i}^{(k, d)}-z_{i}^{(k, d)}\right|<C_{14}\left(A^{*}+A^{* *}+R^{*}\right) k^{-2} .
$$

(b) Now consider the case $z_{0}^{(k, d)}=\omega$. Set $\rho(k)=\frac{C_{15} H}{8 k^{d+2}}$ where $C_{15}$ is to be determined. We again require that $\rho(k)<\frac{C_{6}}{k}$. We have shown that whenever $k>K_{6} A^{*}$, there exists a neighborhood $D_{0}^{(k)}$ containing $\omega$ such that for every $z^{*} \in \partial D_{0}^{(k)}$ we have $\left|p_{k}^{d}\left(z^{*}\right)\right|>C_{16} \rho(k)=\frac{C_{15} C_{16} H}{8 k^{d+2}}$. On the other hand, by (4.13) for every such $z^{*}$ we have

$$
\begin{aligned}
\left|e_{k}^{d}\left(z^{*}\right)\right| & <A^{* *} \times\left(\frac{C_{24}}{k^{d+2}}+\frac{C_{25}}{k^{d+1}} \rho(k)+\frac{C_{26}}{k} \rho^{2}(k)\right)+\frac{C_{19} R^{*}}{k^{d_{1}-d+1}} \\
& <\frac{A^{* *} \times\left(C_{24}+C_{25} C_{6}+C_{26} C_{6}^{2}\right)+C_{19} R^{*}}{k^{d+2}}<\frac{C_{27}\left(A^{* *}+R^{*}\right)}{k^{d+2}} .
\end{aligned}
$$

So we require $\frac{C_{15} C_{16} H}{8 k^{d+2}}>\frac{C_{27}\left(A^{* *}+R^{*}\right)}{k^{d+2}}$ together with $\frac{C_{15} H}{8 k^{d+2}}<\frac{C_{6}}{k}$. This is possible, for example, when

$$
\begin{aligned}
C_{15} & =\frac{8 C_{27}}{C_{16}}, \\
k & >K_{5} H \geq\left(\frac{C_{15} H}{8 C_{6}}\right)^{\frac{1}{d+1}} .
\end{aligned}
$$

Thus we have completed the proof of Lemma 4.12 .

We can finally combine everything and prove the main result of this section.

Theorem 4.13. Let $f$ have $d_{1} \geq 2 d+1$ continuous derivatives everywhere in $[-\pi, \pi] \backslash\{\xi\}$. Let $q_{k}^{d}(z)$ be as defined in (3.3), and let $\left\{\kappa_{i}^{(k, d)}\right\}_{i=0}^{d}$ denote its roots, such that $\left|\kappa_{0}^{(k, d)}\right| \leq \cdots \leq\left|\kappa_{d}^{(k, d)}\right|$. Let $\left\{\phi_{i}\right\}_{i=1}^{d}$ denote the roots of the Laguerre polynomial $\mathcal{L}_{d}^{(1)}$, such that $\left|\phi_{1}\right|<\cdots<\left|\phi_{d}\right|$. Let $y_{0}^{(k, d)}=\omega$ and $y_{i}^{(k, d)}=\mathcal{T}^{-1}\left(-\frac{\phi_{i}}{k}\right)$ for $i=1, \ldots, d$ (see (4.8)). Then there exist constants $C_{9}, C_{15}, C_{28}$ and $K_{9}$ such that for every $k>K_{9} H$ the following statements are true.

(1) The numbers $\left\{y_{i}^{(k, d)}\right\}$ lie on the ray $O \omega$, so that $\left|y_{i}^{(k, d)}\right| \geq 1$, and

$$
C_{9} k^{-1} \leq\left|y_{i}^{(k, d)}-y_{j}^{(k, d)}\right| \quad 0 \leq i<j \leq d .
$$

(2) Each of the numbers $\left\{\kappa_{i}^{(k, d)}\right\}_{i=1}^{d}$ is close to some $y_{i}^{(k, d)}$ :

$$
\left|\kappa_{i}^{(k, d)}-y_{i}^{(k, d)}\right| \leq C_{28} \cdot H \cdot k^{-2} .
$$




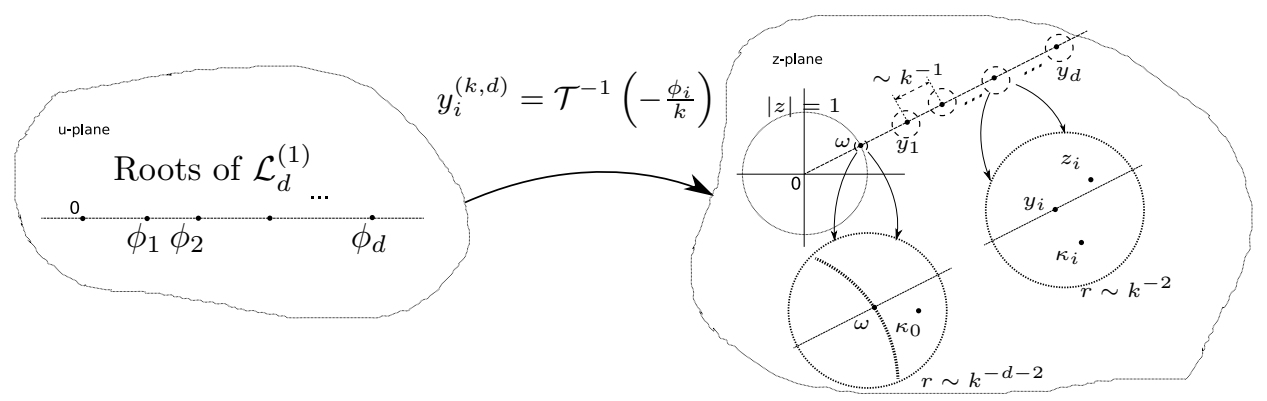

Figure 4.1. The geometry of $\left\{\kappa_{i}^{(k, d)}\right\},\left\{y_{i}^{(k, d)}\right\},\left\{z_{i}^{(k, d)}\right\}$. The superscripts $(k, d)$ are omitted. The picture on the right shrinks towards the unit circle as $k \rightarrow \infty$.

(3) The smallest $\kappa_{0}^{(k, d)}$ is very close to $\omega$ :

$$
\left|\kappa_{0}^{(k, d)}-\omega\right| \leq C_{15} \cdot H \cdot k^{-d-2}
$$

(4) Algorithm 3.2 provides an approximation for $\omega$ which is accurate up to order $k^{-(d+2)}$.

Proof. We have already proved (11) (for $k>K_{2}$ ) and (13) (for $k>K_{3} A^{*}$ ); see Lemma 4.12. (22) follows from Lemma 4.12 and Lemma 4.11 by choosing $C_{28}=C_{14}+C_{11}$ and $k>K_{5} H$. In order to prove (44), we need to show that no root $\kappa_{i}^{(k, d)}$ is closer to the unit circle than $\kappa_{0}^{(k, d)}$. From geometric considerations (see Figure 4.1), it is sufficient to require that

$$
\left|\kappa_{i}^{(k, d)}-y_{i}^{(k, d)}\right| \leq C_{28} \cdot H \cdot k^{-2}<\frac{1}{2} C_{9} k^{-1}<\frac{1}{2} \min _{j \neq i}\left|y_{i}^{(k, d)}-y_{j}^{(k, d)}\right|,
$$

which is true whenever

$$
k>\frac{2 C_{28} H}{C_{9}} .
$$

Therefore we choose

$$
K_{9}=\max \left(K_{2}, K_{3}, K_{5}, \frac{2 C_{28}}{C_{9}}\right) .
$$

4.2. Accuracy analysis: jump magnitudes. Suppose that $d_{1} \geq 2 d+1$ and let $k>K_{9} H$ so that our algorithm gives an approximation $\widetilde{\omega}_{(k)}$ with error at most $C_{15} H \cdot k^{-d-2}$, in accordance with Theorem 4.13. Our goal is to analyze the accuracy of calculating the approximate jump magnitudes $A_{l}^{(k)}$, given by the solution of the linear system (3.5). For convenience, we denote

$$
\begin{aligned}
B_{l} & \stackrel{\text { def }}{=} \imath^{l} A_{d-l}, \\
\widetilde{B}_{l}^{(k)} & \stackrel{\text { def }}{=} \imath^{l} \widetilde{A}_{d-l}^{(k)} .
\end{aligned}
$$


We consider only the case of exactly $d+1$ equations. Thus we can write this system in the following form:

$$
\left[\begin{array}{c}
r_{k} \widetilde{\omega}_{(k)}^{-k} \\
\vdots \\
r_{k+d} \widetilde{\omega}_{(k)}^{-k-d}
\end{array}\right]=V_{k} \times\left[\begin{array}{c}
\widetilde{B}_{0}^{(k)} \\
\vdots \\
\widetilde{B}_{d}^{(k)}
\end{array}\right]
$$

where $V_{k}$ is the $(d+1) \times(d+1)$ system matrix

$$
V_{k} \stackrel{\text { def }}{=}\left[\begin{array}{cccc}
1 & k & \ldots & k^{d} \\
1 & (k+1) & \ldots & (k+1)^{d} \\
\vdots & \vdots & \vdots & \vdots \\
1 & (k+d) & \ldots & (k+d)^{d}
\end{array}\right] .
$$

By (3.4), the "true" coefficients $B_{j}$ satisfy

$$
\left[\begin{array}{c}
m_{k} \omega^{-k} \\
\vdots \\
m_{k+d} \omega^{-k-d}
\end{array}\right]=V_{k} \times\left[\begin{array}{c}
B_{0} \\
\vdots \\
B_{d}
\end{array}\right] .
$$

Our goal is to estimate the error $\varepsilon_{j}^{(k)} \stackrel{\text { def }}{=} B_{j}-\widetilde{B}_{j}^{(k)}$ for $j=0,1, \ldots, d$. Let

$$
\eta_{j}^{(k)} \stackrel{\text { def }}{=} r_{k+j} \widetilde{\omega}_{(k)}^{-k-j}-m_{k+j} \omega^{-k-j} .
$$

Then subtracting (4.16) from (4.15) gives

$$
\left[\begin{array}{c}
\varepsilon_{0}^{(k)} \\
\varepsilon_{1}^{(k)} \\
\vdots \\
\varepsilon_{d}^{(k)}
\end{array}\right]=V_{k}^{-1} \times\left[\begin{array}{c}
\eta_{0}^{(k)} \\
\eta_{1}^{(k)} \\
\vdots \\
\eta_{d}^{(k)}
\end{array}\right]
$$

This is the key relation of this section. In order to estimate the magnitude of $\varepsilon_{j}^{(k)}$, we shall first write out explicit expansions for the quantities $\eta_{j}^{(k)}$, and then investigate how these expansions are transformed when multiplied by the matrix $V_{k}^{-1}$. Our analysis will show that the special combination of the structures of both this matrix and the expansion coefficients results in remarkable cancellations.

Let us start by investigating the structure of the matrix $V_{k}$.

Definition 4.14. Let $S_{k, d}$ denote the $(d+1) \times(d+1)$ square matrix with entries:

$$
\left(S_{k, r}\right)_{m, n}=(-k)^{n-m}\left(\begin{array}{c}
n-1 \\
n-m
\end{array}\right) .
$$

Example 4.15. For $d=4$, we have

$$
S_{k, 4}=\left(\begin{array}{ccccc}
1 & -k & k^{2} & -k^{3} & k^{4} \\
0 & 1 & -2 k & 3 k^{2} & -4 k^{3} \\
0 & 0 & 1 & -3 k & 6 k^{2} \\
0 & 0 & 0 & 1 & -4 k \\
0 & 0 & 0 & 0 & 1
\end{array}\right)
$$

Definition 4.16. For every $k \in \mathbb{N}$ let the symbol $\mathbf{v}_{\mathbf{k}}$ denote the following $1 \times(d+1)$ row vector

$$
\mathbf{v}_{\mathbf{k}} \stackrel{\text { def }}{=}\left[\begin{array}{llll}
1 & k & \ldots & k^{d}
\end{array}\right] .
$$


With this definition, we have

$$
V_{k}=\left[\begin{array}{c}
\mathbf{v}_{\mathbf{k}} \\
\mathbf{v}_{\mathbf{k}+\mathbf{1}} \\
\vdots \\
\mathbf{v}_{\mathbf{k}+\mathbf{d}}
\end{array}\right] .
$$

Lemma 4.17. Let $k \geq 0$, then

$$
V_{k}^{-1}=S_{k, d} \times V_{0}^{-1} .
$$

Proof. Let $1 \leq m \leq d+1$ and $0 \leq t \leq d$. The $m$-th entry of the vector $\mathbf{v}_{\mathbf{k}+\mathbf{t}} \times S_{k, d}$ is equal to

$$
\left(\mathbf{v}_{\mathbf{k}+\mathbf{t}} \times S_{k, d}\right)_{m}=\sum_{l=0}^{m-1}(k+t)^{l}(-k)^{m-1-l}\left(\begin{array}{c}
m-1 \\
m-1-l
\end{array}\right)=t^{m-1},
$$

and therefore

$$
\mathbf{v}_{\mathbf{k}+\mathbf{t}} \times S_{k, d}=\mathbf{v}_{\mathbf{t}} .
$$

(4.19) then follows from (4.18).

Now we would like to expand $\eta_{j}^{(k)}$. We can obviously assume the equality

$$
\widetilde{\omega}_{(k)}=\omega+\frac{\alpha(k)}{k^{d+2}} \quad \text { s.t. } \quad|\alpha(k)| \leq C_{15} H .
$$

Now we estimate $\widetilde{\omega}_{(k)}$ by Proposition $\AA .7$ as follows:

$$
\begin{aligned}
\left(\omega+\frac{\alpha(k)}{k^{d+2}}\right)^{-(k+j)} & =\omega^{-k-j}\left(1+\frac{\alpha(k) \omega^{-1}}{k^{d+2}}\right)^{-k-j} \\
& =\omega^{-k-j}\left(1-(k+j) \frac{\alpha(k) \omega^{-1}}{k^{d+2}}+R_{1}(k, j)\right)
\end{aligned}
$$

where $k$ is large enough so that $\frac{\alpha(k) \omega^{-1}}{k^{d+2}}<\frac{3}{k+j+2}$ is satisfied, and

$$
\left|R_{1}(k, j)\right|<\frac{(k+j)(k+j+1) \alpha^{2}(k) \omega^{-2}}{2 k^{2(d+2)}\left(1-\frac{\alpha(k) \omega^{-1}(k+j+2)}{3 k^{d+2}}\right)}<C_{29} \cdot H^{2} k^{-2 d-3} .
$$

Obviously, $\left|r_{k}\right| \leq C_{30} \cdot H \cdot k^{d}$. Now by (4.12), we have

$$
\begin{aligned}
\eta_{j}^{(k)}= & \left(m_{k+j}+\omega^{k+j} \cdot \sum_{l=1}^{d_{1}-d} \frac{A_{d+l}}{(\imath(k+j))^{l}}+\delta_{k+j}\right) \widetilde{\omega}_{(k)}^{-k-j}-m_{k+j} \omega^{-k-j} \\
= & \left(m_{k+j}+\omega^{k+j} \cdot \sum_{l=1}^{d_{1}-d} \frac{A_{d+l}}{(\imath(k+j))^{l}}+\delta_{k+j}\right) \omega^{-k-j}\left(1-\frac{(k+j) \alpha(k) \omega^{-1}}{k^{d+2}}\right) \\
& -m_{k+j} \omega^{-k-j}+r_{k+j} \omega^{-k-j} R_{1}(k) \\
= & \frac{\beta(k)}{k^{d+2}} \sum_{l=0}^{d} B_{l}(k+j)^{l+1}+\sum_{l=1}^{d_{1}-d} \frac{A_{d+l}}{(\imath(k+j))^{l}}+R_{2}(k, j)
\end{aligned}
$$

where $\left|R_{2}(k, j)\right| \leq C_{31} \cdot H^{2} k^{-d-2}$ and $|\beta(k)| \leq C_{32} \cdot H$. 
Therefore we can write

$$
\begin{aligned}
& {\left[\begin{array}{c}
\eta_{0}^{(k)} \\
\vdots \\
\eta_{j}^{(k)} \\
\vdots \\
\eta_{d}^{(k)}
\end{array}\right]=\beta(k) B_{0}\left[\begin{array}{c}
\frac{k}{k^{d+2}} \\
\vdots \\
\frac{k+j}{k^{d+2}} \\
\vdots \\
\frac{k+d}{k^{d+2}}
\end{array}\right]+\cdots+\beta(k) B_{l}\left[\begin{array}{c}
\frac{k^{l}}{k^{d+2}} \\
\vdots \\
\frac{(k+j)^{l}}{k^{d+2}} \\
\vdots \\
\frac{(k+d)^{l}}{k^{d+2}}
\end{array}\right]+\ldots} \\
& +\beta(k) B_{d}\left[\begin{array}{c}
\frac{k^{d+1}}{k^{d+2}} \\
\vdots \\
\frac{(k+j)^{d+1}}{k^{d+2}} \\
\vdots \\
\frac{(k+d)^{d+1}}{k^{d+2}}
\end{array}\right] \\
& +\frac{A_{d+1}}{\imath}\left[\begin{array}{c}
\frac{1}{k} \\
\vdots \\
\frac{1}{k+j} \\
\vdots \\
\frac{1}{k+d}
\end{array}\right]+\cdots+\frac{A_{d+l}}{\imath^{l}}\left[\begin{array}{c}
\frac{1}{k^{l}} \\
\vdots \\
\frac{1}{(k+j)^{l}} \\
\vdots \\
\frac{1}{(k+d)^{l}}
\end{array}\right]+\ldots \\
& +\frac{A_{d_{1}}}{\imath^{d}}\left[\begin{array}{c}
\frac{1}{k^{d+1}} \\
\vdots \\
\frac{1}{(k+j)^{d+1}} \\
\vdots \\
\frac{1}{(k+d)^{d+1}}
\end{array}\right]+\left[\begin{array}{c}
R_{2}(k, 0) \\
\vdots \\
R_{2}(k, j) \\
\vdots \\
R_{2}(k, d)
\end{array}\right]
\end{aligned}
$$

In light of (4.17), we would now like to examine the action of the matrix $V_{0}^{-1}$ on the vectors on the right-hand side of (4.20).

Lemma 4.18. Let $j=0,1, \ldots, d$.

(1) If $l=1,2, \ldots, d$, then

$$
\left[\begin{array}{c}
k^{l} \\
\vdots \\
(k+j)^{l} \\
\vdots \\
(k+d)^{l}
\end{array}\right]=V_{0} \times\left[\begin{array}{c}
k^{l}\left(\begin{array}{c}
l \\
0
\end{array}\right) \\
\vdots \\
k^{l-j}\left(\begin{array}{l}
l \\
j
\end{array}\right) \\
\vdots \\
1 \cdot\left(\begin{array}{l}
l \\
l
\end{array}\right) \\
0 \\
\vdots \\
0
\end{array}\right] .
$$


(2) Otherwise, there exists a function $R_{3}:\{0,1, \ldots, d\} \rightarrow \mathbb{R}$ such that

$$
\left[\begin{array}{c}
k^{d+1} \\
\vdots \\
(k+j)^{d+1} \\
\vdots \\
(k+d)^{d+1}
\end{array}\right]=V_{0} \times\left[\begin{array}{c}
k^{d+1}\left(\begin{array}{c}
d+1 \\
0
\end{array}\right) \\
\vdots \\
k^{d+1-j}\left(\begin{array}{c}
d+1 \\
j
\end{array}\right) \\
\vdots \\
k\left(\begin{array}{c}
d+1 \\
1
\end{array}\right)
\end{array}\right]+\left[\begin{array}{c}
R_{3}(0) \\
\vdots \\
R_{3}(j) \\
\vdots \\
R_{3}(d)
\end{array}\right] .
$$

Proof. Straightforward application of the binomial theorem.

Lemma 4.19. For $j=1,2, \ldots$ and $i=1, \ldots, d+1$ denote

$$
\tau_{j}^{i} \stackrel{\text { def }}{=}(-1)^{i-1}\left(\begin{array}{c}
j+i-2 \\
j-1
\end{array}\right) .
$$

Then there exists a bounded function $R_{4}:\{0,1, \ldots, d\} \times \mathbb{N} \rightarrow \mathbb{R}$ such that

$$
\left[\begin{array}{c}
\frac{1}{k^{j}} \\
\frac{1}{(k+1)^{j}} \\
\vdots \\
\frac{1}{(k+d)^{j}}
\end{array}\right]=V_{0} \times\left[\begin{array}{c}
\tau_{j}^{1} \\
\frac{\tau_{j}^{j}}{k^{j+1}} \\
\vdots \\
\frac{\tau_{j}^{d+1}}{k^{j+d}}
\end{array}\right]+\frac{1}{k^{d+j+1}}\left[\begin{array}{c}
R_{4}(0, j) \\
\vdots \\
R_{4}(l, j) \\
\vdots \\
R_{4}(d, j)
\end{array}\right]
$$

Proof. First recall the well-known power series expansion 2

$$
\frac{1}{(1+x)^{j}}=\sum_{n=0}^{\infty}(-1)^{n}\left(\begin{array}{c}
j-1+n \\
j-1
\end{array}\right) x^{n} .
$$

Now let $l=0,1, \ldots, d$.

(1) On one hand, the $(l+1)$-st entry in the product on the right-hand side of (4.23) is equal to

$$
g_{j, l} \stackrel{\text { def }}{=} \sum_{i=0}^{d}(-1)^{i} \frac{\left(\begin{array}{c}
j-1+i \\
i
\end{array}\right)}{k^{i+j}} l^{i} .
$$

(2) On the other hand, by Proposition A.7 we have for some bounded function $R_{4}:\{0,1, \ldots, d\} \times \mathbb{N} \rightarrow \mathbb{R}$

$$
\begin{aligned}
\frac{1}{(k+l)^{j}} & =\frac{1}{k^{j}} \cdot \frac{1}{\left(1+\frac{l}{k}\right)^{j}}=\frac{1}{k^{j}}\left\{\sum_{i=0}^{d}(-1)^{i}\left(\begin{array}{c}
j-1+i \\
j-1
\end{array}\right)\left(\frac{l}{k}\right)^{i}+\frac{R_{4}(l, j)}{k^{d+1}}\right\} \\
& =\underbrace{\sum_{i=0}^{d}(-1)^{i} \frac{\left(\begin{array}{l}
j-1+i \\
k^{i+j}
\end{array}\right)}{k^{i}}}_{=g_{j, l}}+\frac{R_{4}(l, j)}{k^{j+d+1}} .
\end{aligned}
$$

Thus (4.23) is proved.

It is now easily seen that the multiplication by $V_{0}^{-1}$ "orders up" the vectors in (4.20) by decreasing powers of $k$. Further multiplication by $S_{k, d}$ from the left preserves this structure, as is evident from the following calculation.

\footnotetext{
2 It can be proven by induction on $j$, using the identity $\sum_{k=0}^{n}\left(\begin{array}{c}r+k \\ r\end{array}\right)=\left(\begin{array}{c}r+n+1 \\ r+1\end{array}\right)$.
} 
Lemma 4.20. Let $c_{i, j}$ be arbitrary constants. Then there exist constants $\gamma_{i, j}$ such that

$$
S_{k, d} \times\left[\begin{array}{c}
\frac{c_{1, j}}{k_{2, j}^{j}} \\
\frac{c_{2, j}^{j}}{k^{j+1}} \\
\vdots \\
\frac{c_{d+1, j}}{k^{j+d}}
\end{array}\right]=\left[\begin{array}{c}
\frac{\gamma_{1, j}}{k^{j}} \\
\frac{\gamma_{2, j}}{k^{j+1}} \\
\vdots \\
\frac{\gamma_{d+1, j}}{k^{j+d}}
\end{array}\right] .
$$

Proof. Let $i=1, \ldots, d+1$ and consider the $i$-th entry of the above product, say $y_{i}$ :

$$
y_{i}=\sum_{l=1}^{d+1}\left(S_{k, d}\right)_{i, l} \times \frac{c_{l, j}}{k^{j+l-1}}=\sum_{l=0}^{d}(-k)^{l+1-i}\left(\begin{array}{c}
l \\
l+1-i
\end{array}\right) \times \frac{c_{l+1, j}}{k^{l+j}}=\frac{1}{k^{j+i-1}} \gamma_{i, j}
$$

where $\gamma_{i, j}=\sum_{l=i-1}^{d}(-1)^{l+1-i}\left(\begin{array}{c}l \\ l-(i-1)\end{array}\right) c_{l+1, j}$. This proves the claim.

We can now prove the main result of this section.

Theorem 4.21. Assume that $d_{1} \geq 2 d+1$ and $k>K_{9} H$, so that by Theorem 4.13 we have $\left|\widetilde{\omega}_{(k)}-\omega\right| \leq C_{15} \cdot H \cdot k^{-d-2}$. Then there exist constants $C_{33}, K_{10}$ such that for every $k>K_{10} H$ and $l=0,1, \ldots, d$ the error in determining $A_{l}$ is

$$
\left|\widetilde{A}_{l}^{(k)}-A_{l}\right| \leq C_{33} \cdot H^{2} \cdot k^{l-d-1} .
$$

Proof. Combine (4.17), (4.20), (4.21), (4.22), (4.23) and (4.24).

\section{LOCALIZING THE DisCONTINUITIES}

As we have seen, both the location and the magnitudes of the jump can be reconstructed with high accuracy. The remaining ingredient in our method is to divide the initial function into regions containing a single jump, and subsequently apply the reconstruction algorithm in each region.

Our approach is to multiply the initial function $f$ by a "bump" $g_{j}$ which vanishes outside some neighborhood of the $j$-th jump. This step requires a priori estimates of the jump positions, which can fortunately be obtained by a variety of methods, for example:

(1) the concentration method of Gelb and Tadmor [22, 18, 41;

(2) the method of partial sums due to Banerjee and Geer [3];

(3) Eckhoff's method with order zero (the Prony method).

All of the above methods provide accurate estimates of $\left\{\xi_{j}\right\}$ up to first order. For definiteness, we present the description of the last method and a rigorous proof of its convergence in Appendix C

Because of the Fourier uncertainty principle, the Fourier series of our bump will have inifinite support and therefore every practically computable convolution will always be an approximation to the exact one. Nevertheless, an error of order at most $k^{-d_{1}-2}$ in the Fourier coefficients will be "absorbed" in the constant $R^{*}$ (4.10) and therefore we will still have accurate estimates for the reconstruction of each separate jump. This will require us to use bump functions which are $C^{\infty}$. An explicit construction of such a function is provided in Appendix B.

We assume that the following quantities are known a priori:

- the lower and upper bounds for the jump magnitudes of order zero: $J_{1} \leq$ $\left|A_{0, j}\right| \leq J_{2}$

- the minimal distance between any two jumps $\left|\xi_{i}-\xi_{j}\right| \geq J_{3}>0$; 
- a constant $T$ for which

$$
\left|2 \pi(\imath k) c_{k}(f)-\sum_{j=1}^{K} A_{0, j} \omega_{j}^{k}\right| \leq T \cdot k^{-1} .
$$

Our localization algorithm can be summarized as follows.

Algorithm 5.1. Let $f$ be a piecewise smooth function of order $d_{1} \geq 2 d+1$ with $K$ jumps $\left\{\xi_{j}\right\}_{j=1}^{K}$ and jump magnitudes $\left\{A_{l, j}\right\}_{l=0, \ldots, d}^{j=1, \ldots, K}$. Let there be given the Fourier coefficients $\left\{c_{k}(f)\right\}_{|k|=0}^{2 M+d+1}$, where $M$ is large enough (see below).

(1) Using the a priori bounds $J_{1}, J_{2}, J_{3}, T$, obtain approximate locations of the jumps $\left\{\widehat{\xi_{j}}\right\}$ via Algorithm C.3. In particular, the error $\left|\widehat{\xi}_{j}-\xi_{j}\right|$ should not exceed $\frac{J_{3}}{3}$, and this will be possible if $M$ is not smaller than required by Theorem C.9.

(2) For each $\widehat{\xi}_{j}$ :

(a) Construct the bump $g_{j}$ centered at $\widehat{\xi_{j}}$ with parameters $t=2 \cdot \frac{J_{3}}{3}$ and $E=J_{3}$, according to Appendix B. Calculate its Fourier coefficients in the range $k=-3 M \ldots 3 M$ according to (B.2).

(b) Now let $h_{j}=f \cdot g_{j}$. For each $k=0,1, \ldots, M+d+1$ calculate

$$
{\widetilde{c_{k}}}^{(M)}\left(h_{j}\right)=\sum_{i=-2 M}^{2 M} c_{i}(f) c_{k-i}\left(g_{j}\right)
$$

(c) Use the above approximate Fourier coefficients $\widetilde{c_{k}}{ }^{(M)}\left(h_{j}\right)$ as the input to Algorithm 3.2 for reconstructing all the parameters of a single jump.

Theorem 5.2. Algorithm 5.1 will produce estimates of all these parameters with the accuracy as stated in Theorems 4.13 and 4.21, $R^{*}$ being replaced with some other constant $\widehat{R^{*}}=\widehat{R^{*}}\left(R^{*}, T, J_{2}, J_{3}\right)$.

Proof. It is clear that the exact function $h_{j}=f \cdot g_{j}$ has exactly one jump at $\xi$ and jump magnitudes $A_{0, j}, \ldots, A_{d, j}$. In order to prove that Theorems 4.13 and 4.21 can be applied, it is sufficient to show that the error $\left|{\widetilde{c_{k}}}^{(M)}\left(h_{j}\right)-c_{k}\left(h_{j}\right)\right|$ is of the order $k^{-\left(d_{1}+2\right)}$. By the Fourier convolution theorem the exact Fourier coefficients of $h_{j}$ are equal to:

$$
c_{k}\left(h_{j}\right)=\sum_{i=-\infty}^{\infty} c_{i}(f) c_{k-i}\left(g_{j}\right)
$$

while our algorithm approximates these by the truncated convolution (5.2). Let us estimate the convolution tail

$$
\Delta c_{k}^{(M)}\left(h_{j}\right)=\sum_{i=-\infty}^{-2 M} c_{i}(f) c_{k-i}\left(g_{j}\right)+\sum_{i=2 M}^{\infty} c_{i}(f) c_{k-i}\left(g_{j}\right) .
$$

On one hand, the Fourier coefficients of $f$ can be bounded using (5.1):

$$
\left|c_{k}(f)\right| \leq C_{34}\left(J_{2}+T\right) k^{-1} \text {. }
$$

On the other hand, taking $\alpha=d_{1}+1$ we have by Theorem B.1.

$$
\left|c_{k}\left(g_{j}\right)\right| \leq \frac{C_{35}}{J_{3}^{d_{1}+1}} \cdot \frac{1}{k^{d_{1}+2}} .
$$


Finally,

$$
\begin{aligned}
\left|\Delta c_{k}^{(M)}\left(h_{j}\right)\right| \leq \frac{C_{36}\left(J_{2}+T\right)}{J_{3}^{d_{1}+1}} \sum_{i=2 M}^{\infty} \frac{1}{i^{d_{1}+3}} & \leq \frac{C_{36}\left(J_{2}+T\right)}{J_{3}^{d_{1}+1}} \zeta\left(d_{1}+3,2 M\right) \\
& \leq \frac{C_{37} \cdot\left(J_{2}+T\right)}{J_{3}^{d_{1}+1}} M^{-d_{1}-2}
\end{aligned}
$$

where $\zeta(s, q)$ is the Hurwitz zeta function. Therefore Algorithm 3.2 will produce estimates of $\left\{\widetilde{\xi}_{j}\right\}$ and $\left\{\widetilde{A}_{l, j}\right\}$ with accuracy as guaranteed by Theorems 4.13 and 4.21 where

$$
\widehat{R^{*}}=R^{*}+\frac{\left(J_{2}+T\right)}{J_{3}^{d_{1}+1}} .
$$

\section{Final aCCuracy}

In this section we are going to calculate the overall accuracy of approximation. Let us therefore suppose that $d_{1} \geq 2 d+1$, and so using the Fourier coefficients $c_{-2 M}(f), \ldots, c_{2 M}(f)$ we have reconstructed the singular part $\Phi(x)$ with accuracy specified by Theorem 5.2. Recall from (2.3) that our final approximation is defined by

$$
\widetilde{f}=\sum_{|k| \leq M}\left(c_{k}(f)-c_{k}(\widetilde{\Phi})\right) \mathrm{e}^{\imath k x}+\widetilde{\Phi}
$$

where $\widetilde{\Phi}=\sum_{j=1}^{K} \sum_{l=0}^{d} \widetilde{A}_{l, j} V_{l}\left(x ; \widetilde{\xi}_{j}\right)$. Intuitively, the approximation error function $\tilde{f}-f$ will look as depicted in Figure 6.1-very small almost everywhere except in some shrinking neighborhoods of the jump points. Let $y \in[-\pi, \pi] \backslash\left\{\xi_{j}\right\}_{j=1}^{K}$. If we take $M$ large enough so that the error estimate of Theorem 4.13 will be less than the distance to the nearest jump $\left|y-\xi_{j}\right|$, then $y$ will lie in the "flat" region of Figure 6.1 and the error $|\tilde{f}(y)-f(y)|$ will be small. This is precisely the content of our final theorem.

Theorem 6.1. Let $f:[-\pi, \pi] \rightarrow \mathbb{R}$ have $K$ jump discontinuities $\left\{\xi_{j}\right\}_{j=1}^{K}$, and let it be $d_{1}$-times continuously differentiable between the jumps. Let $r>0$. Then for every integer d satisfying $2 d+1 \leq d_{1}$, there exist explicit functions $F=F\left(\mathbb{A}, \widehat{R^{*}}\right), G=$ $G\left(\mathbb{A}, \widehat{R^{*}}, r\right)$ depending on all of the a priori bounds such that for all $M>G$ Algorithm 2.2 reconstructs the locations and the magnitudes of the jumps with accuracy provided by Theorem 5.2, and with the following pointwise accuracy in the "jump-free region":

$$
|\widetilde{f}(y)-f(y)| \leq F \cdot M^{-d-1} \quad y \in[-\pi, \pi] \backslash\left(\bigcup_{j=1}^{K} B_{r}\left(\xi_{j}\right)\right) .
$$

Proof. Define

$$
f_{M} \stackrel{\text { def }}{=} \sum_{|k| \leq M}\left(c_{k}(f)-c_{k}(\Phi)\right) \mathrm{e}^{\imath k x}+\Phi .
$$

We write the overall approximation error as

$$
|\widetilde{f}(y)-f(y)| \leq\left|\widetilde{f}(y)-f_{M}(y)\right|+\left|f_{M}(y)-f(y)\right| .
$$




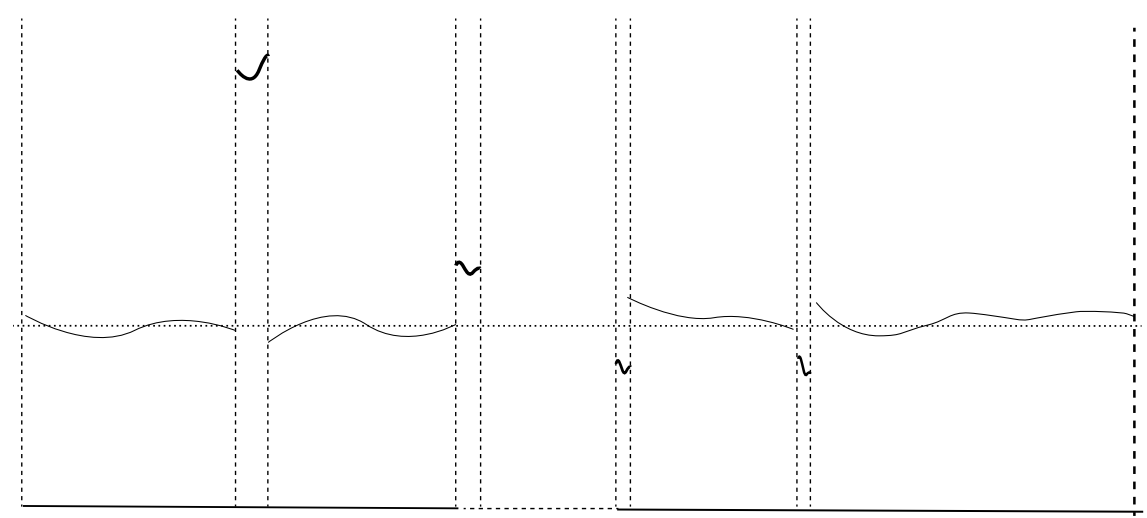

FiguRE 6.1. The approximation error

Let us examine the two terms on the right-hand side separately.

According to our previous notation, $\Psi=f-\Phi$ is a $d$-times continuously differentiable everywhere function. Therefore, using (2.5) we have the estimate

$$
\begin{aligned}
\left|f_{M}(y)-f(y)\right| & =\left|\sum_{|k| \leq M} c_{k}(\Psi) \mathrm{e}^{\imath k y}-\Psi(y)\right| \\
& =\left|\sum_{|k|>M} c_{k}(\Psi) \mathrm{e}^{\imath k y}\right| \leq C_{38} \cdot R \cdot M^{-d-1} .
\end{aligned}
$$

Let $\Theta \stackrel{\text { def }}{=} \widetilde{\Phi}-\Phi$ denote the "singular error function" (see Figure 6.1). Then the second term in (6.1) can be written as:

$$
\begin{aligned}
\left|\widetilde{f}(y)-f_{M}(y)\right| & =\left|\sum_{|k| \leq M}\left(c_{k}(\Phi)-c_{k}(\widetilde{\Phi})\right) \mathrm{e}^{\imath k y}+(\widetilde{\Phi}(y)-\Phi(y))\right| \\
& =\left|\sum_{|k| \leq M} c_{k}(\Theta) \mathrm{e}^{\imath k y}-\Theta(y)\right| .
\end{aligned}
$$

Write

$$
\begin{array}{rr}
\widetilde{\xi}_{j}=\xi_{j}+\alpha(M) & |\alpha(M)| \leq F_{\alpha}\left(A^{*}, A^{* *}, \widehat{R^{*}}\right) \cdot M^{-d-2}, \\
\widetilde{A}_{l, j}=A_{l, j}+\beta_{l}(M) & \left|\beta_{l}(M)\right| \leq F_{\beta}\left(A^{*}, A^{* *}, \widehat{R^{*}}\right) \cdot M^{l-d-1}
\end{array}
$$

where $F_{\alpha}$ and $F_{\beta}$ are provided by Theorem 5.2 For every $\epsilon<r$, we define

$$
U_{l, \epsilon}(y) \stackrel{\text { def }}{=} V_{l}\left(y ; \xi_{j}+\epsilon\right)-V_{l}\left(y ; \xi_{j}\right) .
$$


Using the formula (2.1) we therefore have

$$
\begin{aligned}
\Theta(y) & =\sum_{j=1}^{K} \sum_{l=0}^{d}\left\{\widetilde{A}_{l, j} V_{l}\left(y ; \widetilde{\xi}_{j}\right)-A_{l, j} V_{l}\left(y ; \xi_{j}\right)\right\} \\
& =\underbrace{\sum_{j=1}^{K} \sum_{l=0}^{d} \beta_{l}(M) V_{l}\left(y ; \xi_{j}\right)}_{\stackrel{\text { def }}{=} Z(y)}+\underbrace{}_{\stackrel{\text { def }_{=} W(y)}{\sum_{j=1}^{K} \sum_{l=0}^{d} \widetilde{A}_{l, j} U_{l, \alpha(M)}(y)},},
\end{aligned}
$$

and so

$$
\left|\widetilde{f}(y)-f_{M}(y)\right| \leq\left|\sum_{|k|>M} c_{k}(Z) \mathrm{e}^{\imath k y}\right|+\left|\sum_{|k|<M} c_{k}(W) \mathrm{e}^{\imath k y}-W(y)\right| .
$$

The functions $V_{l}$ belong to $C^{l}$, and therefore by the well-known estimate (see also (1.1)), there exist constants $S_{l}$ such that

$$
\left|\sum_{|k|>M} c_{k}\left(V_{l}\right) \mathrm{e}^{\imath k y}\right| \leq S_{l} \cdot M^{-l}
$$

and therefore

$$
\left|\sum_{|k|>M} c_{k}(Z) \mathrm{e}^{\imath k y}\right| \leq C_{39} \cdot F_{\beta} \cdot M^{-d-1} .
$$

Let us now investigate $W(y)$. Let $L$ denote an upper bound for the magnitudes of the jumps:

$$
\left|A_{l, j}\right|<L \quad j=1, \ldots, K ; \quad l=0,1, \ldots, d_{1} .
$$

Clearly, the functions $U_{l, \epsilon}(y)$ satisfy the following properties.

(1) $\left|U_{l, \epsilon}(y)\right| \leq C_{40} \epsilon$ for some absolute constant $C_{40}$ (recall that $y$ is in the "jump-free region"). This bound can be obtained by just Taylor-expanding the functions $V_{l}\left(y ; \xi_{j}+\epsilon\right)$ at $\epsilon=0$. In particular, for $\epsilon=\alpha(M)$ we have

$$
|W(y)| \leq \sum_{j=1}^{K} \sum_{l=0}^{d}\left|\widetilde{A}_{l, j}\right|\left|U_{l, \alpha(M)}(y)\right| \leq C_{41} \cdot L \cdot F_{\alpha} \cdot M^{-d-2} .
$$

(2) In the "no man's land" of length $\alpha(M)$ between $\xi_{j}$ and $\widetilde{\xi}_{j}, U_{l, \epsilon}$ is bounded by $C_{42} \cdot L$. Furthermore, as we have just seen, in the flat regions $U_{l, \alpha(M)}$ is bounded by $C_{40} F_{\alpha} M^{-d-2}$. Therefore the Fourier coefficients of $W$ are certainly bounded by

$$
\left|c_{k}(W)\right| \leq C_{43} \cdot L \cdot F_{\alpha} \cdot M^{-d-2}
$$

and so

$$
\left|\sum_{|k|<M} c_{k}(W) \mathrm{e}^{\imath k y}\right| \leq C_{44} \cdot L \cdot F_{\alpha} \cdot M^{-d-1} .
$$

Combining (6.1), (6.2), (6.3), (6.4), (6.5) and (6.6) completes the proof. 


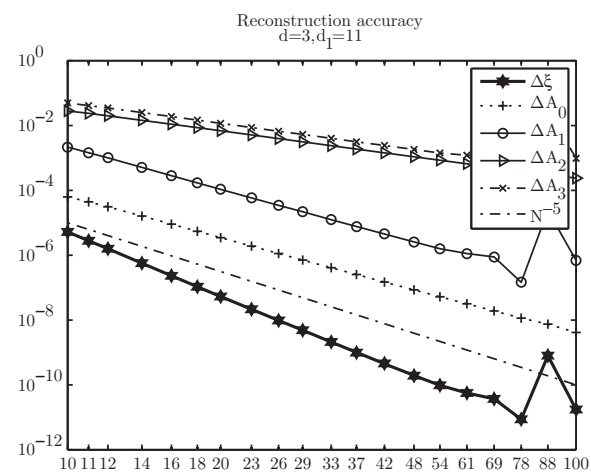

(A) Accuracy of reconstruction with $d=3$ and $d_{1}=11$, as a function of $M$.

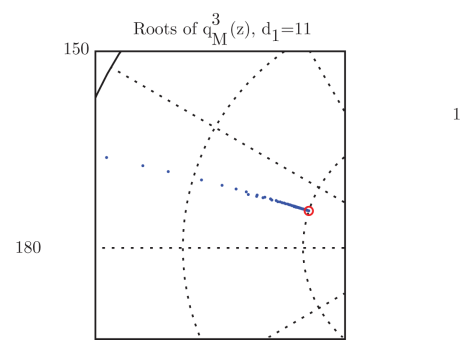

(B) The roots of $q_{M}^{d}(z)$

FiguRE 7.1. Reconstruction of a single jump

\section{Numerical Results}

In this section we present results of various numerical simulations whose primary goal is to validate the asymptotic accuracy predictions for large $M$. We have used a straightforward implementation and made no attempt to optimize it further. In particular, the Fourier coefficients are assumed to be known with arbitrary precision (this is important for the localization, see below).

7.1. Recovery of a single jump. Given $d, d_{1}$ and $M$, a piecewise function with one discontinuity is generated according to the formula

$$
f(x)=\sum_{l=0}^{d_{1}} A_{l} V_{l}(x ; \xi)+\sum_{k=-M}^{M} f_{k} \mathrm{e}^{\imath k x}
$$

where the numbers $\xi \in[-\pi, \pi],\left\{A_{l} \in \mathbb{R}_{l=0}^{d_{1}}\right.$ and $\left\{f_{k} \in \mathbb{C}\right\}_{k=-M}^{M}$ are chosen at random, such that $f_{k} \sim k^{-d_{1}-2}$ and $f_{-k}=\bar{f}_{k}$. The Fourier coefficients are calculated with the exact formula

$$
c_{k}(f)=\frac{\mathrm{e}^{-\imath k \xi}}{2 \pi} \sum_{l=0}^{d_{1}} \frac{A_{l}}{(\imath k)^{l+1}}+f_{k} .
$$

These coefficients are then passed to the reconstruction routine for a single jump, of order $d$. This routine implements Algorithm 3.2 in a standard MATLAB environment with double-precision calculations.

The following experiments were carried out.

(1) Keeping $d$ and $d_{1}$ fixed, we compared the accuracy of recovering the jump location and all the jump magnitudes for different values of $M$. The results can be seen in Figure 7.1. We also plot the distribution of roots of the corresponding polynomials $q_{M}^{d}(z)$; compare with Figure 4.1.

(2) Keeping $d_{1}$ fixed, we compared the accuracy for different reconstruction orders $d=1, \ldots, d_{1}$. The results are presented in Figure 7.2 .

(3) Keeping the reconstruction order $d$ fixed, we compared the accuracy for different smoothness values $d_{1}$. The results are presented in Figure 7.3 . 


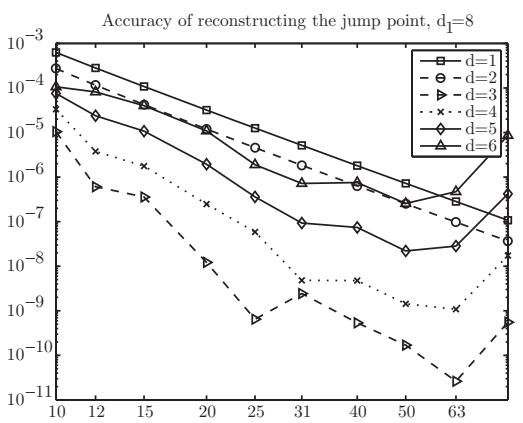

(A) $d_{1}=8$

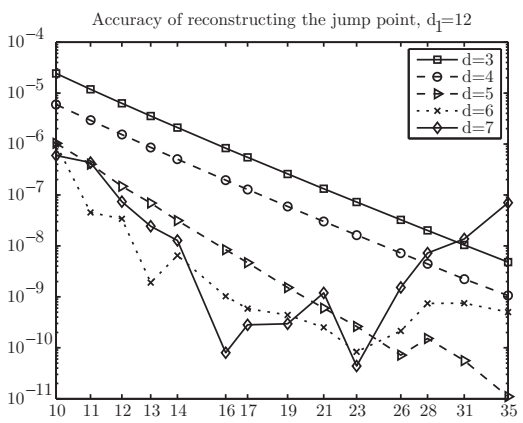

(B) $d_{1}=12$

FiguRE 7.2. Dependence of the accuracy on the order with fixed smoothness, with increasing $M$.

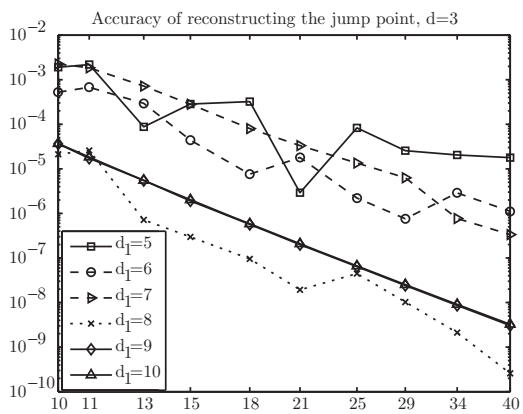

(A) $d=3$

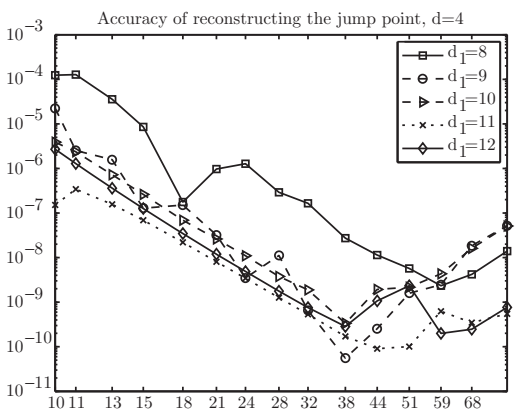

(B) $d=4$

FiguRE 7.3. Dependence of the accuracy on the smoothness with fixed order, with increasing $M$.

The optimality of $d=\frac{d_{1}}{2}-1$, as well as the asymptotic order of convergence, are clearly seen to fit the theoretical predictions. The instability and eventual breakup of the measured accuracy for large values of $M$ is due to the finite-precision calculations.

7.2. Localization. We have restricted ourselves to the following simplified setting: the function has two jumps at $\xi_{1}=0$ and $\xi_{2}=3$, and we localize the jump at the origin by a bump having width $\frac{8}{3}$ around the initial approximation $\widehat{\xi_{1}}=\frac{1}{40}$. The explicit formulas for the Fourier coefficients of the bump are derived in Appendix B, We have used Mathematica in order to carry out the computations with arbitrary precision.

The results can be seen in Figure 7.4, Localization convergence can clearly be seen here, although it starts from very large coefficients. This seems to suggest that although the asymptotic accuracy order is correct, more research is required in order for the proposed localization procedure (i.e. Fourier convolution) to be suitable for practical applications. In particular, the various constants appearing in the bounds of Section 5 need to be carefully analyzed. 


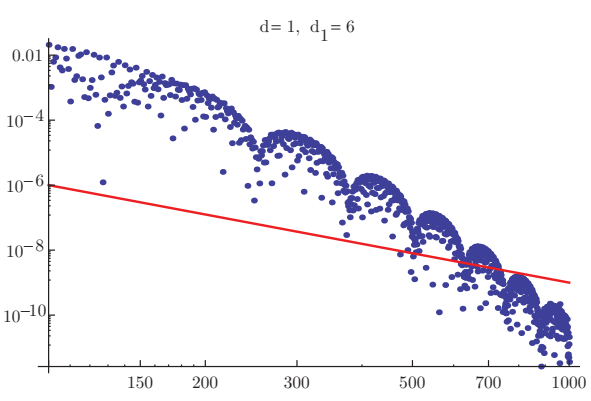

(A) The case $d=1, d_{1}=6$

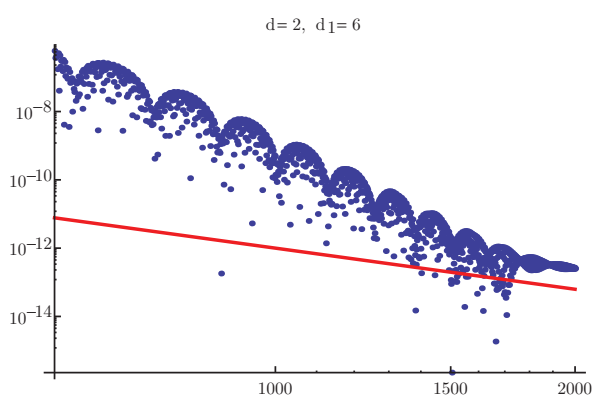

(B) The case $d=2, d_{1}=6$

FiguRE 7.4. Localization: accuracy of recovering the jump. The predicted accuracy $M^{-d-2}$ is drawn for comparison.

\section{Discussion}

In this paper we have demonstrated that nonlinear Fourier reconstruction of piecewise smooth functions can achieve accuracy with asymptotic order of at least half the order of smoothness. As indicated by our theoretical results as well as the numerical simulations, a reconstruction method whose order is more than half the order of smoothness becomes less accurate. So it appears that the algebraic approach has certain limitations, and the interesting question is whether these limitations are inherent or superficial. We hope that our results may provide a clue towards obtaining sharp upper bounds.

From the practical point of view, the one-jump case seems to be promising as we have good convergence for moderate values of $M$. While the multiple-jump case needs further investigation in order to attain similar results, in the end it may prove beneficial, since in this case we would not need to know a priori the number of jumps but still have high resolution in the well-separated regions, by successively applying the localization each time.

In addition, it seems that Eckhoff's conjecture is false as stated in 15, namely that the jumps of a piecewise smooth $C^{d}$ function can be reconstructed with accuracy $k^{-d-2}$. Using a method of highest possible order doesn't take into account the stiffness of the problem. In fact, it can be shown that the Lipschitz constant of the solution map $\left\{c_{k}(f)\right\}_{k=M}^{M+d+1} \rightarrow\left\{\xi_{j}, A_{l, j}\right\}$ of order $d$ is proportional to $M^{d}$. We plan to present these results elsewhere.

Hopefully, our analysis can be related to the algebraic reconstruction schemes of Kvernadze and Banerjee and Geer as well.

We would like to point out the connection of the algebraic system (2.2) as well as the well-known Prony system of equations (C.1) (which plays a central role in many branches of mathematics, see [37] and [32]) to other recent nonlinear reconstruction methods in Signal Processing, in particular: finite rate of innovation techniques [42, 12, reconstruction of shapes from moments [26, 23] and piecewise $D$-finite moment inversion 6, [7. We therefore hope that our results can be extended to these subjects as well. 


\section{Appendix A. Miscellaneous auxiliary Related Results}

In this appendix we provide proofs of several combinatorial auxiliary results.

Let E denote the discrete "shift" operator in $k$, i.e., for every function $g(k)$ : $\mathbb{R} \rightarrow \mathbb{R}$ we have

$$
\mathrm{E} g(k) \stackrel{\text { def }}{=} g(k+1)
$$

Furthermore, let $\Delta$ denote the discrete difference operator, i.e., $\Delta=\mathrm{E}-\mathrm{I}$ where I is the identity operator. Then by the binomial theorem we have

$$
\Delta^{d} g(k)=(\mathrm{E}-\mathrm{I})^{d} g(k)=(-1)^{d} \sum_{j=0}^{d}(-1)^{j}\left(\begin{array}{l}
d \\
j
\end{array}\right) g(k+j) \text {. }
$$

Lemma A.1. Let $p(k)=a_{0} k^{n}+a_{1} k^{n-1}+\cdots+a_{n}$ be a polynomial of degree $n$. Then

$$
\begin{aligned}
\Delta^{n} p(k) & =a_{0} n !, \\
\Delta^{n+1} p(k) & =0 .
\end{aligned}
$$

Proof. See e.g. 17.

Now assume that $g(k): \mathbb{R}^{+} \rightarrow \mathbb{R}$ is a given function. Let us perform a change of variable $y=\frac{1}{k}$, and define $G(y) \stackrel{\text { def }}{=} g\left(\frac{1}{y}\right)=g(k)$. With this notation, we have

$$
\Delta g(k)=g(k+1)-g(k)=g\left(\frac{1}{y}+1\right)-g\left(\frac{1}{y}\right)=G\left(\frac{y}{1+y}\right)-G(y) .
$$

We subsequently define the "dual" operator $\mathcal{D}$ as:

$$
\mathcal{D}\{G(y)\} \stackrel{\text { def }}{=} G\left(\frac{y}{1+y}\right)-G(y) \text {. }
$$

The operator $\mathcal{D}$ has an interesting property of "killing" the lowest-order Taylor coefficient at 0 .

Proposition A.2. Let $H(y)$ be analytic at $y=0$, such that $H(y)=h_{m} y^{m}+$ $h_{m+1} y^{m+1}+\ldots$ Then for $n \in \mathbb{N}$, the function $\mathcal{D}^{n}\{H(y)\}$ is analytic at 0 with Taylor expansion

$$
\mathcal{D}^{n}\{H(y)\}=h_{m+n}^{*} y^{m+n}+h_{m+n+1}^{*} y^{m+n+1}+\ldots .
$$

Proof. The proof is by induction on $n$. The basis $n=0$ is given. Assuming that $U(y)=\mathcal{D}^{n-1}\{H(y)\}$ is analytic at 0 and $U(y)=u_{m+n-1}^{*} y^{m+n-1}+u_{m+n-1}^{*} y^{m+n-1}+$ $\ldots$, consider the function $\mathcal{D}\{U(y)\}=\mathcal{D}^{n}\{H(y)\}$. Let $z=\frac{y}{1+y}$, and so for $|y|<1$ we have

$$
z(y)=y\left(1-y+y^{2}+\ldots\right)=y-y^{2}+\ldots .
$$

Making the analytic change of coordinates $y \rightarrow z(y)$ we conclude that the Taylor expansion of $U(z(y))$ at the origin is

$$
U(z(y))=u_{m+n-1}^{*} z^{m+n-1}+\cdots=u_{m+n-1}^{*} y^{m+n-1}+\ldots .
$$

That is, the leading coefficient is the same as in the Taylor expansion of $U(y)$. Therefore

$$
\begin{aligned}
\mathcal{D}\{U(y)\} & =U(z)-U(y) \\
& =u_{m+n}^{* *} y^{m+n}+u_{m+n+1}^{* *} y^{m+n+1}+\ldots .
\end{aligned}
$$

This expansion holds in some neighborhood of the origin. 
Lemma A.3. Let $l, d \in \mathbb{N}$. Then there exist positive constants $C_{45}, K_{11}$ such that for all $k>K_{11}$,

$$
\left|\sum_{j=0}^{d}(-1)^{j}\left(\begin{array}{l}
d \\
j
\end{array}\right) \frac{1}{(k+j)^{l}}\right|<\frac{C_{45}}{k^{d+l}} .
$$

Proof. Let $g(k)=\frac{1}{k^{l}}$. It is easy to check (see (A.1)) that

$$
A_{l, d}(k) \stackrel{\text { def }}{=} \sum_{j=0}^{d}(-1)^{j}\left(\begin{array}{l}
d \\
j
\end{array}\right) \frac{1}{(k+j)^{l}}=\Delta^{d} g(k) .
$$

The proof of the claim is in two steps. First, we shall develop the expression $A_{l, d}(k)$ into power series in $\frac{1}{k}$ converging for sufficiently large $k$. Then, based on this representation we shall establish the required bound.

Let $y=\frac{1}{k}$. According to our notation, let $G(y)=g\left(\frac{1}{y}\right)=g(k)$ and so $A_{l, d}(k)=$ $\mathcal{D}^{d}\{G(y)\} \stackrel{\text { def }}{=} F(y)$. Furthermore, for all $j=0,1, \ldots$ we have

$$
k+j=\frac{1}{y}+j=\frac{1+j y}{y}
$$

and so

$$
F(y)=\sum_{j=0}^{d}(-1)^{j}\left(\begin{array}{l}
d \\
j
\end{array}\right) G\left(\frac{y}{1+j y}\right) .
$$

Substituting $G(y)=y^{l}$, we conclude that $F(y)$ is a real analytic function of $y$ in the disk $|y|<\frac{1}{d}$, and so it can be written as a converging power series

$$
F(y)=\sum_{i=0}^{\infty} f_{i} y^{i}
$$

Applying Proposition $A .2$ to $G(y)$ we conclude that $f_{0}=\cdots=f_{l+d-1}=0$. Therefore the expansion

$$
A_{l, d}(k)=\sum_{i=l+d}^{\infty} \frac{f_{i}}{k^{i}}
$$

holds for $k>d$.

Let us now estimate the magnitude of the coefficients $f_{i}$. Since (A.2) is valid for $k=d+1$, then there exists a constant $C_{46}$ such that $\left|f_{i}(d+1)^{-i}\right|<C_{46}$ for all $i \in \mathbb{N}$ and therefore

$$
\left|f_{i}\right|<C_{46}(d+1)^{i} .
$$

But then for arbitrary $k \geq d+2$ we have

$$
\begin{aligned}
\left|A_{l, d}(k)\right| & =\left|\sum_{i=0}^{\infty} \frac{f_{l+d+i}}{k^{l+d+i}}\right|<\frac{C_{46}(d+1)^{l+d}}{k^{l+d}} \sum_{i=0}^{\infty} \frac{(d+1)^{i}}{k^{i}} \\
& \leq \frac{C_{46}(d+1)^{l+d}}{k^{l+d}} \cdot \frac{1}{1-\frac{d+1}{d+2}} \leq \frac{C_{45}}{k^{l+d}} .
\end{aligned}
$$


Lemma A.4. Let $\omega \in \mathbb{C}, a_{0}, \ldots, a_{n} \in \mathbb{C}$ and $n, k \in \mathbb{N}$. Denote $b_{k} \stackrel{\text { def }}{=} \omega^{k}$. $\left(a_{0}+a_{1} k+\cdots+a_{n} k^{n}\right)$. Then

$$
\sum_{j=0}^{n+1}(-1)^{j}\left(\begin{array}{c}
n+1 \\
j
\end{array}\right) b_{k+j} \omega^{n+1-j} \equiv 0 .
$$

Proof. Denote

$$
p(k) \stackrel{\text { def }}{=} a_{0}+a_{1} k+\ldots a_{n} k^{n} .
$$

Then we rewrite the given expression as

$$
\begin{aligned}
\sum_{j=0}^{n+1}(-1)^{j}\left(\begin{array}{c}
n+1 \\
j
\end{array}\right) b_{k+j} \omega^{n+1-j} & =\sum_{j=0}^{n+1}(-1)^{j}\left(\begin{array}{c}
n+1 \\
j
\end{array}\right) p(k+j) \omega^{n+k+1} \\
& =(-1)^{d+1} \omega^{n+k+1} \Delta^{n+1} p(k) . \\
\text { (Lemma A.1 } & =0 .
\end{aligned}
$$

The claim is therefore proved.

Theorem A.5 (Rouche's theorem). Let the polynomial $q(z) \in \mathbb{C}[z]$ be a sum $q(z)=$ $p(z)+e(z)$. Let $z_{0}$ be a simple zero of $p(z)$. If there exists $\rho \in \mathbb{R}^{+}$such that

$$
|p(z)|>|e(z)| \quad \forall z \in \partial B_{\rho}\left(z_{0}\right)
$$

then $q(z)$ has a simple zero inside $B_{\rho}\left(z_{0}\right)$.

Lemma A.6. Let there be given a sequence of polynomials $P_{k}(z): \mathbb{C} \rightarrow \mathbb{C}$ and $a$ point $z_{0} \in \mathbb{C}$ such that

(1) $P_{k}\left(z_{0}\right)=0$ for all $k \in \mathbb{N}$;

(2) $\left|P_{k}^{\prime}\left(z_{0}\right)\right| \geq C_{47}$ for all $k \in \mathbb{N}$ and some constant $C_{47}$ independent of $k$;

(3) for every fixed $k$ the following inequality holds for all $z \in B_{k^{-1}}\left(z_{0}\right)$,

$$
\left|P_{k}^{\prime \prime}(z)\right| \leq C_{48} k
$$

where $C_{48}$ is a constant independent of $k$.

Let $\rho(k): \mathbb{N} \rightarrow \mathbb{R}$ satisfy

$$
0<\rho(k)<\min \left(\frac{1}{k}, \frac{C_{47}}{C_{48} k}\right) .
$$

Then there exists a constant $C_{49}$ independent of $\rho(k)$ such that for all $k$, the following holds for every $z \in \partial B_{\rho(k)}\left(z_{0}\right)$ :

$$
\left|P_{k}(z)\right| \geq C_{49} \rho(k)
$$

Proof. Let us write the truncated Taylor expansion of $P_{k}$ around $z_{0}$ with remainder in Lagrange form:

$$
P_{k}\left(z_{0}+\rho(k) e^{\imath \theta}\right)=P_{k}\left(z_{0}\right)+\underbrace{P_{k}^{\prime}\left(z_{0}\right) \rho(k) \mathrm{e}^{\imath \theta}}_{=E_{1}}+\underbrace{\frac{P_{k}^{\prime \prime}(\xi)}{2} \rho^{2}(k) \mathrm{e}^{2 \imath \theta}}_{=E_{2}}
$$

for some $\xi \in B_{\rho}\left(z_{0}\right)$. Now since $\rho(k) \leq \frac{1}{k}$ we have

$$
\begin{aligned}
& \left|E_{1}\right| \geq C_{47} \rho(k), \\
& \left|E_{2}\right| \leq \frac{C_{48} k \rho^{2}(k)}{2} .
\end{aligned}
$$


On the other hand,

$$
\begin{aligned}
\rho(k) & \leq \frac{C_{47}}{C_{48} k}, \\
\frac{C_{48} k \rho^{2}(k)}{2} & \leq \frac{C_{47} \rho(k)}{2} .
\end{aligned}
$$

Therefore $\frac{\left|E_{1}\right|}{2} \geq\left|E_{2}\right|$ and so by taking $C_{49} \stackrel{\text { def }}{=} \frac{C_{47}}{2}$ we have $\left|P_{k}(z)\right| \geq C_{49} \rho(k)$.

Proposition A.7. Let $n \in \mathbb{N}$ be given. Then for $|x|<\frac{3}{n+2}$ the following estimate holds:

$$
(1+x)^{-n}=1-n x+\frac{n(n+1)}{2} x^{2} R_{5}(x) \quad \text { where } \quad\left|R_{5}(x)\right|<\frac{1}{1-\frac{x(n+2)}{3}} .
$$

In general, for approximation of order $d$, we have for $|x|<\frac{d+2}{n+d+1}$,

$$
\begin{aligned}
(1+x)^{-n}=1 & -n x+\frac{n(n+1)}{2} x^{2}+\cdots+(-1)^{d} \frac{n \times \cdots \times(n+d-1)}{d !} x^{d} \\
& +(-1)^{d+1} \frac{n \times \cdots \times(n+d)}{(d+1) !} x^{d+1} R_{6}(x)
\end{aligned}
$$

where

$$
\left|R_{6}(x)\right|<\frac{1}{1-\frac{(n+d+1)}{d+2} x} .
$$

Proof. Standard majorization of the Taylor series tail by a geometric series.

\section{Appendix B. Explicit CONSTRUCtion of A Bump}

In this appendix we present an explicit construction of the bump function which we used in our numerical simulations. We also derive an explicit bound for the size of its Fourier coefficients to be used in the proof of localization accuracy.

Let there be given two parameters $t$ and $E$ with $2 E>t$, together with the point $\xi \in \mathbb{R}$. Our goal is to build a function $g=g_{E, t}(x ; \xi)$ which satisfies the following conditions:

(G1) $g \equiv 0$ for $x \notin[\xi-E, \xi+E]$;

(G2) $g \equiv 1$ for $x \in\left[\xi-\frac{t}{2}, \xi+\frac{t}{2}\right]$;

(G3) $g \in C^{\infty}(\mathbb{R})$;

(G4) the Fourier coefficients of $g$ decay as rapidly as possible.

The idea is to take a standard $C^{\infty}$ mollifier, scale it and convolve with a box function.

We define two new parameters: the scaling factor $s$ and the width of the box $r$. Note that our construction implies $r \geq 2 s$, because otherwise the result of the convolution will not have a flat segment in the middle.

Let us therefore take the standard $C^{\infty}$ mollifier

$$
\Psi(x)= \begin{cases}\mathrm{e}^{-1 /\left(1-x^{2}\right)} & \text { for }|x|<1, \\ 0 & \text { otherwise, }\end{cases}
$$

and scale it between $-s$ and $s$ for some $s>0$ :

$$
m_{s}(x)=\frac{1}{s \Delta} \Psi\left(\frac{x}{s}\right)
$$


where

$$
\Delta=\frac{1}{s} \int_{-s}^{s} \Psi\left(\frac{x}{s}\right) \mathrm{d} x=\int_{-1}^{1} \Psi(y) \mathrm{d} y \sim 0.443994 .
$$

Now we take a box function centered at $\xi$, having width $r$ :

$$
b_{r}(x ; \xi)= \begin{cases}1 & \text { for }-\frac{r}{2} \leq x-\xi \leq \frac{r}{2} \\ 0 & \text { otherwise }\end{cases}
$$

Finally, we convolve the two and get a smooth bump:

$$
g=g_{r, s}(x ; \xi)=b_{r}(x ; \xi) * m_{s}(x)=\frac{1}{s \Delta} \int_{\xi-\frac{r}{2}}^{\xi+\frac{r}{2}} \Psi\left(\frac{x-t}{s}\right) \mathrm{d} t .
$$

The new parameters $s, r$ should be compatible with the original $E, t$. In particular, we want to have a strip of width $t$ in the center, and the extent of the whole bump should not exceed $E$. Therefore we have the following compatibility conditions:

$$
\begin{aligned}
s+\frac{t}{2} & <\frac{r}{2}, \\
2 s+\frac{r}{2} & <E .
\end{aligned}
$$

The function $g$ so constructed, clearly satisfies conditions (G1)-(G3) above. Let us now maximize the decay of its Fourier coefficients. By definition:

$$
c_{k}(g)=\frac{1}{2 \pi s \Delta} \int_{-\pi}^{\pi} \mathrm{e}^{-\imath k x}\left\{\int_{\xi-\frac{r}{2}}^{\xi+\frac{r}{2}} \Psi\left(\frac{x-t}{s}\right) \mathrm{d} t\right\} \mathrm{d} x .
$$

Notice first that $\Psi(z)$ is zero outside the region $-1 \leq z \leq 1$, therefore we can make the change of variables $z \rightarrow t-x, t \rightarrow t$ and rewrite the integral as

$$
c_{k}(g)=\frac{1}{2 \pi s \Delta} \int_{-s}^{s} \mathrm{e}^{\imath k z} \Psi\left(\frac{z}{s}\right)\left\{\int_{\xi-\frac{r}{2}}^{\xi+\frac{r}{2}} \mathrm{e}^{-\imath k t} \mathrm{~d} t\right\} \mathrm{d} z .
$$

So now the two integrals are completely separated. Explicit calculation gives

$$
\int_{\xi-\frac{r}{2}}^{\xi+\frac{r}{2}} \mathrm{e}^{-\imath k t} \mathrm{~d} t=-\frac{\imath \mathrm{e}^{-\frac{1}{2} \imath k(r+2 \xi)}\left(-1+\mathrm{e}^{\imath k r}\right)}{k} .
$$

Now we scale back: $z=s y$ and obtain the explicit formula

$$
\begin{aligned}
c_{k}(g) & =-\frac{\imath \mathrm{e}^{-\frac{1}{2} \imath k(r+2 \xi)}\left(-1+\mathrm{e}^{\imath k r}\right)}{k} \cdot \frac{1}{2 \pi \Delta} \int_{-1}^{1} \mathrm{e}^{\imath k s y} \Psi(y) \mathrm{d} y \\
& =-\frac{\imath \mathrm{e}^{-\frac{1}{2} \imath k(r+2 \xi)}\left(-1+\mathrm{e}^{\imath k r}\right)}{2 \pi \Delta k} c_{-k s}(\Psi) .
\end{aligned}
$$

Finally, we would like to determine the optimal values for $s$ and $r$ so that $\left|c_{k}(g)\right|$ decrease as rapidly as possible with $k \rightarrow \infty$.

First note that since $\Psi \in C^{\infty}$, then for every $\alpha>1$ there exists a constant $C_{50}(\alpha)$ such that

$$
\left|c_{k}(\Psi)\right| \leq C_{50} \cdot|k|^{-\alpha}
$$




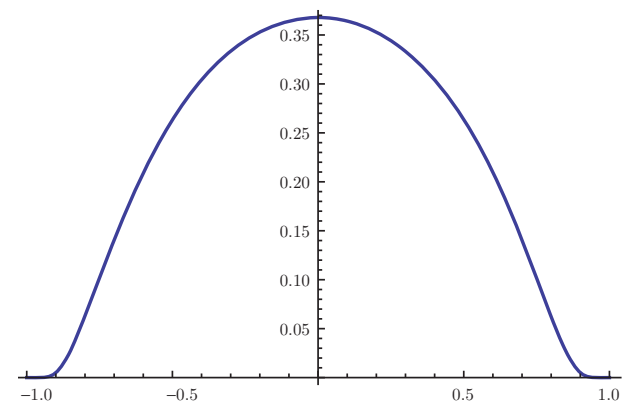

(A) The standard mollifier $\Psi(x)$

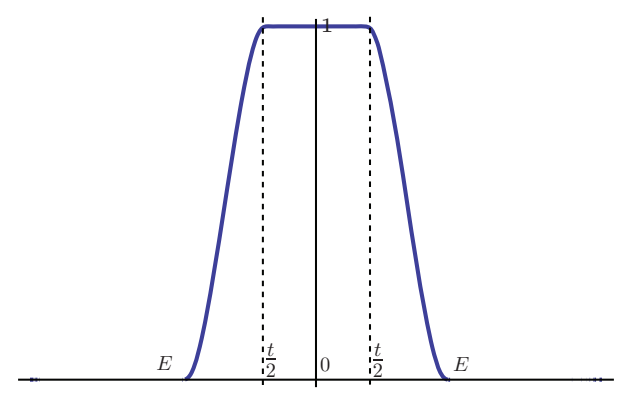

(B) The final bump for $\xi=0$

Figure B.1. The construction of the bump

The formula (B.2) suggests that we should take $s$ to be as large as possible. Applying the conditions (B.1) we get that the following values maximize $s$ :

$$
\begin{aligned}
s^{*} & =\frac{1}{3}\left(E-\frac{t}{2}\right), \\
r^{*} & =\frac{2}{3}(E+t) .
\end{aligned}
$$

We have thus proved the following result:

Theorem B.1. Given $E, t$ with $2 E>t$ and a point $\xi$, let $g_{E, t}(x ; \xi)$ be the bump constructed above. Then it satisfies the conditions (G1)-(G4) such that for every $\alpha>1$ there exists a constant $C_{51}=C_{51}(\alpha)$ such that for all $k \in \mathbb{N}$,

$$
\left|c_{k}\left(g_{E, t}\right)\right| \leq C_{51} \cdot(2 E-t)^{-\alpha} k^{-1-\alpha} \text {. }
$$

\section{Appendix C. Initial estimates Via Prony's method}

In this appendix we present a rigorous proof that the Eckhoff's method of order zero produces sufficiently accurate estimates of the jump locations $\left\{\xi_{j}\right\}$, to be used in Algorithm 5.1. Denote $\omega_{j}=\mathrm{e}^{-\imath \xi_{j}}$. For $d=0$, the system (2.2) becomes

$$
\underbrace{\sum_{j=1}^{K} A_{0, j} \omega_{j}^{k}}_{=m_{k}} \approx 2 \pi(\imath k) c_{k}(f) .
$$

(C.1) is a well-known system of equations which is sometimes called the Prony system ([37]) or Sylvester-Ramanujan system ([32]). The original method of solution (due to Baron de Prony, [36]) is to exploit the following fact.

Lemma C.1. The sequence $\left\{m_{k}\right\}$ satisfies the recurrence relation with constant coefficients

$$
\sum_{i=0}^{K} m_{k+i} q_{i}=0
$$

where $\left\{q_{i}\right\}$ are the coefficients of the polynomial

$$
Q(z) \stackrel{\text { def }}{=} \prod_{j=1}^{K}\left(z-\omega_{j}\right)=\sum_{i=0}^{K} q_{i} z^{i} .
$$


Proof. We have $Q\left(\omega_{j}\right)=0$ for all $j=1, \ldots, K$. Therefore

$$
\sum_{i=0}^{K} q_{i} m_{k+i}=\sum_{i=0}^{K} q_{i} \sum_{j=1}^{K} A_{0, j} \omega_{j}^{k+i}=\sum_{j=1}^{K} A_{0, j} \omega_{j}^{k} \sum_{i=0}^{K} q_{i} \omega_{j}^{i}=\sum_{j=1}^{K} A_{0, j} \omega_{j}^{k} Q\left(\omega_{j}\right)=0 .
$$

Corollary C.2. Let $q_{K}=1$ for normalization. Then for all $k \in \mathbb{N}$ the coefficient vector $\left\{q_{i}\right\}_{i=0}^{K-1}$ is the solution of the linear system

$$
\underbrace{\left[\begin{array}{cccc}
m_{k} & m_{k+1} & \cdots & m_{k+K-1} \\
m_{k+1} & m_{k+2} & \cdots & m_{k+K} \\
\vdots & \vdots & \vdots & \vdots \\
m_{k+K-1} & m_{k+K} & \cdots & m_{k+2 K-2}
\end{array}\right]}_{\stackrel{\text { def }}{=} H_{k}} \times\left[\begin{array}{c}
q_{0} \\
q_{1} \\
\vdots \\
q_{K-1}
\end{array}\right]=-\left[\begin{array}{c}
m_{k+K} \\
m_{k+K+1} \\
\vdots \\
m_{k+2 K-1}
\end{array}\right]
$$

After this preparation, we can now describe the algorithm for obtaining initial estimates using Prony's method. Recall that our a priori bounds are given by $J_{1}, J_{2}, J_{3}$ and $T$; see Section 5 .

Algorithm C.3. Let us be given the first $M+2 K-1$ Fourier coefficients $c_{k}(f)$ of a function $f$ with $K$ unknown discontinuities $\left\{\xi_{j}\right\}_{j=1}^{K}$, which is continuously differentiable between these discontinuities. Denote the magnitudes of the jumps by $\left\{A_{j}\right\}_{j=1}^{K}$.

(1) Calculate the sequence

$$
r_{k}=2 \pi(\imath k) c_{k}(f)
$$

(2) Solve the system

$$
\underbrace{\left[\begin{array}{cccc}
r_{M} & r_{M+1} & \cdots & r_{M+K-1} \\
r_{M+1} & r_{M+2} & \cdots & r_{M+K} \\
\vdots & \vdots & \vdots & \vdots \\
r_{M+K-1} & r_{M+K} & \cdots & r_{M+2 K-2}
\end{array}\right]}_{\stackrel{\text { def }}{=} \widetilde{H_{M}}} \times\left[\begin{array}{c}
\widetilde{q}_{0} \\
\widetilde{q}_{1} \\
\vdots \\
\widetilde{q}_{K-1}
\end{array}\right]=-\left[\begin{array}{c}
r_{M+K} \\
r_{M+K+1} \\
\vdots \\
r_{M+2 K-1}
\end{array}\right] .
$$

(3) Take the estimated $\left\{\widetilde{\omega}_{j}\right\}$ to be the roots of the polynomial

$$
\widetilde{Q}(z)=z^{K}+\sum_{i=0}^{K-1} \widetilde{q}_{i} z^{i}
$$

and then set

$$
\widetilde{\xi}_{j}=-\arg \widetilde{\omega}_{j} .
$$

Now we would like to analyze the accuracy of Algorithm C.3. First, we need to estimate the error in solving the system (C.3). We use standard result from numerical linear algebra.

Lemma C.4. Consider the linear system $A x=b$ and let $\mathbf{x}_{\mathbf{0}}$ be the exact solution. Let this system be perturbed:

$$
(A+\Delta A) x=b+\Delta b
$$


and let $x_{0}+\Delta x$ denote the exact solution of this perturbed system. Denote

$$
\delta x=\frac{\|\Delta x\|}{\left\|x_{0}\right\|} \quad \delta A=\frac{\|\Delta A\|}{\|A\|} \quad \delta b=\frac{\|\Delta b\|}{\|b\|} \quad \kappa=\|A\|\left\|A^{-1}\right\| \text { (condition number) }
$$

for some vector norm $\|\cdot\|$ and the induced matrix norm. Then

$$
\delta x \leq \frac{\kappa}{1-\kappa \cdot \delta A}(\delta A+\delta b) .
$$

Proof. See e.g. 45].

Consider (C.3). The error in the right-hand side is given by (5.1). Therefore we now need to estimate the condition number of the matrix $H_{k}$. Although all the entries are bounded, it may still happen 3 that $\kappa\left(H_{k}\right)$ is unbounded. Fortunately, this is not the case. To see this, we are going to factorize $H_{k}$ into a component which depends on $k$, and a component which doesn't.

Lemma C.5. Let $V=V\left(\xi_{1}, \ldots, \xi_{K}\right)$ denote the Vandermonde matrix on the nodes $\left\{\omega_{j}\right\}$, i.e.,

$$
V=\left[\begin{array}{cccc}
1 & 1 & \ldots & 1 \\
\omega_{1} & \omega_{2} & \ldots & \omega_{K} \\
\vdots & \vdots & \ddots & \vdots \\
\omega_{1}^{K-1} & \omega_{2}^{K-1} & \ldots & \omega_{K}^{K-1}
\end{array}\right]
$$

Then for all $k \in \mathbb{N}$,

$$
H_{k}=V \times \operatorname{diag}\left\{A_{0, j} \omega_{j}^{k}\right\} \times V^{T} .
$$

Proof. Direct computation from the definitions (C.1) and (C.2).

Corollary C.6. For all $k \in \mathbb{N}$,

$$
\kappa\left(H_{k}\right) \leq \frac{J_{2}}{J_{1}} \kappa^{2}(V) .
$$

Remark C.7. $\kappa(V)$ is well studied in e.g. [20]. It essentially depends on the minimal distance between the nodes. In particular,

$$
\left\|V^{-1}\right\| \sim \max _{1 \leq i \leq K} \prod_{j=1, j \neq i}^{n} \frac{1}{\left|\omega_{j}-\omega_{i}\right|}
$$

Lemma C.8. There exist constants $C_{52}, K_{12}$ such that for all $i=0,1, \ldots, d$ and for all $k>K_{12} \frac{T J_{2}}{J_{1}^{2}} \kappa^{2}(V)$,

$$
\left|q_{i}-\widetilde{q}_{i}\right| \leq C_{52} \frac{T J_{2}}{J_{1}^{2}} \kappa^{2}(V) k^{-1}
$$

Proof. In the context of Lemma C.4 our original system is $H_{k} \mathbf{q}=\mathbf{m}$ (C.2) and the perturbed system is $\widetilde{H}_{k} \widetilde{\mathbf{q}}=\widetilde{\mathbf{m}}$ C.3). Note that $\left|m_{k}\right| \geq J_{1} \cdot C_{53}$ for some $C_{53}$.

$$
{ }^{3} \text { Consider, for instance, } H_{k}=\left[\begin{array}{cc}
1 & 1+\frac{1}{k} \\
1 & 1-\frac{1}{k}
\end{array}\right] \text {. }
$$


From previous considerations we therefore have

$$
\begin{aligned}
\delta H_{k} & =\frac{\left\|\widetilde{H}_{k}-H_{k}\right\|}{\left\|H_{k}\right\|} \leq C_{54} \frac{T}{J_{1}} \cdot \frac{1}{k}, \\
\delta \mathbf{m} & \leq C_{55} \cdot \frac{T}{J_{1}} \cdot \frac{1}{k}, \\
\kappa\left(H_{k}\right) & \leq \frac{J_{2}}{J_{1}} \kappa^{2}(V) .
\end{aligned}
$$

We would like to estimate $\delta \mathbf{q}$ according to (‥4). If

$$
k>\underbrace{2 C_{54}}_{\stackrel{\text { def }}{=} K_{12}} \frac{T}{J_{1}} \cdot \frac{J_{2}}{J_{1}} \kappa^{2}(V),
$$

then $\kappa^{2}\left(H_{k}\right) \delta H_{k} \leq \frac{1}{2}$ and so

$$
\delta \mathbf{q} \leq \underbrace{J_{2}}_{\stackrel{\text { def }}{=} C_{52}\left(C_{54}+C_{55}\right)} \kappa_{1}^{2}(V) \frac{T}{J_{1}} \cdot \frac{1}{k} .
$$

We can finally estimate the accuracy of Algorithm C.3. To shorten notation, let

$$
F=F(\mathbb{A}, \mathbb{G}, T) \stackrel{\text { def }}{=} \frac{T J_{2}}{J_{1}^{2}} \kappa^{2}(V) .
$$

Theorem C.9. For every $0<\alpha<1$ there exist constants $C_{56}(\alpha), K_{13}(\alpha, F)$ such that for every $k>K_{13}$ Algorithm C.3 reconstructs the locations of the jumps with accuracy

$$
\left|\xi_{j}-\widetilde{\xi}_{j}\right| \leq C_{56} \cdot F \cdot k^{\alpha-1} .
$$

Proof. We have shown that the perturbation $Q-\widetilde{Q}$ has coefficients of magnitude $k^{-1}$. We will use the same reasoning as in Section 4 in order to estimate the quantity $\left|\omega_{j}-\widetilde{\omega}_{j}\right|$, which is the perturbation of the roots of $Q(z)$. Let $|z|<2$. Since the coefficients of $Q(z)$ do not depend on $k$, we can obviously find constants $C_{57}$ and $C_{58}$ such that

(1) $\left|Q^{\prime}\left(\omega_{j}\right)\right| \geq C_{57}$,

(2) $\left|Q^{\prime \prime}(z)\right|<C_{58}$.

By reasoning similar to Lemma A.6 we conclude that there exist constants $C_{59}<$ $1, C_{60}$ such that for every function $0<\rho(k)<C_{59}$ we have

$$
|Q(z)|>C_{60} \rho(k) \quad \forall z \in \partial B_{\rho(k)}\left(\omega_{j}\right) .
$$

Now let

$$
\rho(k)=F \cdot k^{\alpha-1} .
$$

If $k>\left(\frac{F}{C_{59}}\right)^{\frac{1}{1-\alpha}}$, then $\rho(k)<C_{59}$ and so $($ C.5 holds. On the other hand, by Lemma C.8 we have that

$$
|(Q-\widetilde{Q})(z)| \leq C_{61} \cdot F \cdot k^{-1}
$$


Now finally we require that $k>\left(\frac{C_{61}}{C_{60}}\right)^{\frac{1}{\alpha}}$, in which case

$$
|(Q-\widetilde{Q})(z)| \leq C_{61} \cdot F \cdot k^{-1}<C_{60} F k^{\alpha-1}<|Q(z)|
$$

and therefore $\widetilde{Q}(z)$ has a simple zero inside $B_{\rho(k)}\left(\omega_{j}\right)$.

Thus we have shown that $\left|\widetilde{\omega}_{j}-\omega_{j}\right| \leq F \cdot k^{\alpha-1}$. Write $\widetilde{\omega}_{j}=\omega_{j}+\beta(k) k^{\alpha-1}$ where $|\beta(k)|<F$. Then by Taylor expansion of the logarithm we will have (recall $\left.\left|\omega_{j}\right|=1\right)$ for large enough $k>K_{13}(\alpha)$,

$$
\left|\widetilde{\xi}_{j}-\xi_{j}\right|=\left|\arg \omega_{j}-\arg \widetilde{\omega}_{j}\right|=\left|\arg \left(\frac{\omega_{j}}{\widetilde{\omega}_{j}}\right)\right| \leq C_{56}(\alpha) \cdot F \cdot k^{\alpha-1} .
$$

\section{REFERENCES}

[1] M. Abramowitz and I.A. Stegun. Handbook of Mathematical Functions with Formulas, Graphs, and Mathematical Tables. National Bureau of Standards Applied Math. Ser., 55, U.S. Govt. Printing Office, Washington, D. C., 1964. MR0167642 (29:4914)

[2] F. Arandiga, A. Cohen, R. Donat, and N. Dyn. Interpolation and approximation of piecewise smooth functions. SIAM Journal on Numerical Analysis, 43:41, 2005. MR2177955 (2006h:41002)

[3] N.S. Banerjee and J.F. Geer. Exponentially accurate approximations to periodic Lipschitz functions based on Fourier series partial sums. Journal of Scientific Computing, 13(4):419460, 1998. MR1676752(2000b:65020)

[4] A. Barkhudaryan, R. Barkhudaryan, and A. Poghosyan. Asymptotic behavior of Eckhoff's method for Fourier series convergence acceleration. Analysis in Theory and Applications, 23(3):228-242, 2007. MR2350105 (2010e:42002)

[5] P. Barone and R. March. Reconstruction of a piecewise constant function from noisy Fourier coefficients by Padé method. SIAM Journal on Applied Mathematics, 60(4):1137-1156, 2000. MR:1760030 (2001b:41018)

[6] D. Batenkov. Moment inversion problem for piecewise D-finite functions. Inverse Problems, 25(10):105001, October 2009. MR 2545970 (2011a:44011)

[7] D. Batenkov, N. Sarig, and Y. Yomdin. An "algebraic" reconstruction of piecewisesmooth functions from integral measurements. Proc. of Sampling Theory and Applications (SAMPTA), 2009.

[8] R. Bauer. Band filters for determining shock locations. PhD thesis, Department of Applied Mathematics, Brown University, Providence, RI, 1995.

[9] B. Beckermann, A.C. Matos, and F. Wielonsky. Reduction of the Gibbs phenomenon for smooth functions with jumps by the $\varepsilon$-algorithm. Journal of Computational and Applied Mathematics, 219(2):329-349, 2008. MR2441229 (2009h:41011)

[10] John P. Boyd. Acceleration of algebraically-converging fourier series when the coefficients have series in powers of 1/n. Journal of Computational Physics, 228(5):1404-1411, 2009. MR2494223 (2010b:65311)

[11] C. Brezinski. Extrapolation algorithms for filtering series of functions, and treating the Gibbs phenomenon. Numerical Algorithms, 36(4):309-329, 2004. MR.2108182 (2005g:42005)

[12] P.L. Dragotti, M. Vetterli, and T. Blu. Sampling moments and reconstructing signals of finite rate of innovation: Shannon meets Strang-Fix. IEEE Transactions on Signal Processing, 55(5):1741, 2007. MR2472334 (2010f:94150)

[13] T.A. Driscoll and B. Fornberg. A Padé-based algorithm for overcoming the Gibbs phenomenon. Numerical Algorithms, 26(1):77-92, 2001. MR1827318(2002b:65007)

[14] K.S. Eckhoff. Accurate and efficient reconstruction of discontinuous functions from truncated series expansions. Mathematics of Computation, 61(204):745-763, 1993. MR1195430 (94a:65073)

[15] K.S. Eckhoff. Accurate reconstructions of functions of finite regularity from truncated Fourier series expansions. Mathematics of Computation, 64(210):671-690, 1995. MR1265014 (95f:65234)

[16] K.S. Eckhoff. On a high order numerical method for functions with singularities. Mathematics of Computation, 67(223):1063-1088, 1998. MR 1459387 (98j:65014) 
[17] S. Elaydi. An Introduction to Difference Equations. Springer, 2005. MR2128146 (2005j:39001)

[18] S. Engelberg and E. Tadmor. Recovery of edges from spectral data with noise - a new perspective. SIAM Journal on Numerical Analysis, 46(5):2620-2635, 2008. MR2421050 (2009d:42005)

[19] B. Ettinger, N. Sarig, and Y. Yomdin. Linear versus non-linear acquisition of step-functions. Journal of Geometric Analysis, 18(2):369-399, 2008. MR2393265 (2009e:41056)

[20] W. Gautschi. Norm estimates for inverses of Vandermonde matrices. Numerische Mathematik, 23(4):337-347, 1974. MR0378383 (51:14550)

[21] A. Gelb and D. Cates. Segmentation of Images from Fourier Spectral Data. Commun. Comput. Phys., 5:326-349, 2009. MR2513689 (2010c:65263)

[22] A. Gelb and E. Tadmor. Detection of edges in spectral data. Applied and Computational Harmonic Analysis, 7(1):101, 1999. MR1699594 (2000g:42003)

[23] G.H. Golub, P. Milanfar, and J. Varah. A stable numerical method for inverting shape from moments. SIAM Journal on Scientific Computing, 21(4):1222-1243, 2000. MR1740393 (2000m:65048)

[24] D. Gottlieb and C.W. Shu. On the Gibbs phenomenon and its resolution. SIAM Review, pages 644-668, 1997. MR1491051 (98m:42002)

[25] C. Guilpin, J. Gacougnolle, and Y. Simon. The $\varepsilon$-algorithm allows to detect Dirac delta functions. Applied Numerical Mathematics, 48(1):27-40, 2004. MR2027820 (2004j:65011)

[26] B. Gustafsson, C. He, P. Milanfar, and M. Putinar. Reconstructing planar domains from their moments. Inverse Problems, 16(4):1053-1070, 2000. MR1776483 (2001k:44010)

[27] T. Hrycak and K. Gröchenig. Pseudospectral Fourier reconstruction with the modified inverse polynomial reconstruction method. Journal of Computational Physics, 229(3):933-946, 2010. MR2566372 (2010k:65313)

[28] L.V. Kantorovich and V.I. Krylov. Approximate Methods of Higher Analysis. Fizmatgiz, Moscow, 1962. MR0154389 (27:4338)

[29] G. Kvernadze. Approximating the jump discontinuities of a function by its Fourier-Jacobi coefficients. Mathematics of Computation, 73(246):731-752, 2004. MR2031403 (2004j:42026)

[30] G. Kvernadze. Approximation of the discontinuities of a function by its classical orthogonal polynomial Fourier coefficients. Math. Comp, 79:2265-2285, 2010. MR2684364

[31] Yaron Lipman and David Levin. Approximating piecewise-smooth functions. IMA J. Numer. Anal. 30(4):1159-1193, 2010. MR2727820

[32] Y.I. Lyubich. The Sylvester-Ramanujan system of equations and the complex power moment problem. The Ramanujan Journal, 8(1):23-45, 2004. MR2068428 (2005e:30068)

[33] H.N. Mhaskar and J. Prestin. Polynomial frames for the detection of singularities. In Wavelet Analysis and Multiresolution Methods: Proceedings of the Conference Held at University of Illinois at Urbana-Champaign, Illinois, page 273. CRC, 2000. MR.1777997(2001k:65212)

[34] I.P. Natanson. Constructive Function Theory (in Russian). Gostekhizdat, 1949.

[35] L. Plaskota and G.W. Wasilkowski. The power of adaptive algorithms for functions with singularities. Journal of Fixed Point Theory and Applications, 6(2):227-248, 2009. MR2580977 (2011b:65269)

[36] R. Prony. Essai experimental et analytique. J. Ec. Polytech.(Paris), 2:24-76, 1795.

[37] N. Sarig and Y. Yomdin. Signal acquisition from measurements via non-linear models. Mathematical Reports of the Academy of Science of the Royal Society of Canada, 29(4):97-114, 2008. MR2426609 (2009h:62029)

[38] B.D. Shizgal and J.H. Jung. Towards the resolution of the Gibbs phenomena. Journal of Computational and Applied Mathematics, 161(1):41-65, 2003. MR2018574 (2004k:42042)

[39] A. Solomonoff. Reconstruction of a discontinuous function from a few Fourier coefficients using Bayesian estimation. Journal of Scientific Computing, 10(1):29-80, 1995. MR.1337448 (96b:65013)

[40] G. Szegő. Orthogonal Polynomials. Colloq. Publ. Vol. 23, American Mathematical Society, 1975. MR0372517(51:8724)

[41] E. Tadmor. Filters, mollifiers and the computation of the Gibbs phenomenon. Acta Numerica, 16:305-378, 2007. MR2417931 (2009c:65033)

[42] M. Vetterli, P. Marziliano, and T. Blu. Sampling signals with finite rate of innovation. IEEE Transactions on Signal Processing, 50(6):1417-1428, 2002. MR.1930786 (2003i:94019)

[43] J. Vindas. Local Behavior of Distributions and Applications. PhD thesis, 2009. 
[44] M. Wei, A.G. Martínez, and A.R. De Pierro. Detection of edges from spectral data: New results. Applied and Computational Harmonic Analysis, 22(3):386-393, 2007. MR2311863 (2008j:42002)

[45] J.H. Wilkinson. Rounding errors in algebraic processes. Dover Publ, 1994. MR.1280465

[46] A. Zygmund. Trigonometric Series. Vols. I, II. Cambridge University Press, New York, 1959. MR0236587 (38:4882)

Department of Mathematics, Weizmann Institute of Science, Rehovot 76100, Israel

E-mail address: dima.batenkov@weizmann.ac.il

URL: http://www.wisdom. weizmann.ac.il/〜dmitryb

Department of Mathematics, Weizmann Institute of Science, Rehovot 76100, Israel

E-mail address: yosef .yomdin@weizmann.ac.il

URL: http://www.wisdom.weizmann.ac.il/ yomdin 